\title{
Average entropy of a subsystem over a global unitary orbit of a mixed bipartite state
}

\author{
Lin Zhang $^{1 *}$ and Hua Xiang ${ }^{2 \dagger}$ \\ ${ }^{1}$ Institute of Mathematics, Hangzhou Dianzi University, Hangzhou 310018, PR China \\ ${ }^{2}$ School of Mathematics and Statistics, Wuhan University, Wuhan 430072, PR China
}

\begin{abstract}
We investigate the average entropy of a subsystem within a global unitary orbit of a given mixed bipartite state in the finite-dimensional space. Without working out the closed-form expression of such average entropy for the mixed state case, we provide an analytical lower bound for this average entropy. In deriving this analytical lower bound, we get some useful by-products of independent interest. We also apply these results to estimate average correlation along a global unitary orbit of a given mixed bipartite state. When the notion of von Neumann entropy is replaced by linear entropy, the similar problem can be considered also, and moreover the exact average linear entropy formula is derived for a subsystem over a global unitary orbit of a mixed bipartite state.
\end{abstract}

Keywords: quantum state; unitary orbit; average entropy; average correlation; Page's formula

\section{Introduction}

In 1978, Lubkin [15] proposed a method of approximating the average entropy for a subsystem of a finitedimensional quantum system in a global pure bipartite state by expanding the entropy as a series in terms of the average traces of powers of the system's reduced density operator, but the convergence of this series was never established. However, the author of recent paper [6] found an exact closed form expression for the average traces, in which Dyer gave a characterization of the convergence of the series.

In fact, Page conjectured in [18] that if a quantum system of Hilbert space dimension $m n$ is in a random pure bipartite state, the average entropy of a subsystem of dimension $m \leqslant n$ should be given by the simple

\footnotetext{
*E-mail: godyalin@163.com; linyz@zju.edu.cn

†E-mail: hxiang@whu.edu.cn
} 
and elegant formula

$$
S_{m, n}=H_{m n}-H_{n}-\frac{m-1}{2 n},
$$

where $H_{k}:=\sum_{j=1}^{k} \frac{1}{j}$ is the $k$-th harmonic number. The average entropy $S_{m, n}$ in (1.1) is also served as a way of understanding the information in black hole radiation. This formula was first proved by Foong and Kanno [7] by using Fourier transform, and next by Sánchez-Ruiz [20] and by Sen [19] by using random matrix theory connected with generalized Laguerre polynomials. Some years later, Lachal [14] used a probabilistic approach to give a re-derivation of Page's formula. Recently, Zhang [23] has shown that if a quantum system of Hilbert space dimension $m n$ is in a random pure bipartite state, the average diagonal entropy of a subsystem of dimension $m \leqslant n$ should be given by the simple and elegant formula

$$
S_{m, n}^{D}=H_{m n}-H_{n}
$$

Based on the above mentioned formulas, i.e. (1.1) and (1.2), he derives quickly that the average coherence of a subsystem of dimension $m \leqslant n$ is given by $S_{m, n}^{D}-S_{m, n}=\frac{m-1}{2 n}$ (see also [26] for another approach).

We know that a random pure state can be generated by a unitary operator chosen uniformly according to Haar measure $\mu$. Thus the above problem can be equivalently described as follows: Consider a complex quantum system $A B$ which consists of two subsystems $A$ and $B$. For a given pure state $\rho_{A B}=\left|\psi_{A B}\right\rangle\left\langle\psi_{A B}\right|$,

$$
\int_{\mathrm{U}\left(d_{A} d_{B}\right)} \mathrm{S}\left(\operatorname{Tr}_{B}\left(U \rho_{A B} U^{\dagger}\right)\right) \mathrm{d} \mu(U)=H_{d_{A} d_{B}}-H_{d_{B}}-\frac{d_{A}-1}{2 d_{B}} \quad\left(d_{A} \leqslant d_{B}\right),
$$

where $S(\rho):=-\operatorname{Tr}(\rho \ln \rho)$ is the von Neumann entropy. Along this line, in a very recent paper [3], Christandl et al computed exactly the eigenvalue distributions of reduced density matrices of multipartite pure state by employing symplectic geometric method. Here we ask: can one have an analogous formula for a given mixed bipartite state $\rho_{A B}$ ? This question has been paid no or little attention to the best of our knowledge. In this note, we will make an attempt to determine the average entropy of a subsystem along a global unitary orbit of a given mixed bipartite state.

In fact, recently, many researchers studied various problems along a unitary orbit of a quantum state. For example, the total correlation attained between the subsystems of a bipartite quantum system is constrained if the bipartite system undergoes global unitary evolutions. The authors of Ref [11, 12] investigated related problems motivated by some considerations in the field of quantum thermodynamics. They have not only obtained the value of the maximal quantum mutual information (QMI), but also the maximum QMI state in the balanced bipartite quantum systems. Unlike the maximum QMI case, finding the minimum QMI state on the unitary orbit is more difficult than finding the maximum QMI state in general. Luckily, they completely solved this minimum QMI state for two-qubit case. Besides, Zhang and Fei investigated relative entropy and fidelity between two unitary orbits of two states, respectively [24]. They have obtained a lot of compact expressions for some extremal values under consideration. We have already known that any bipartite quantum state can be diagonalized under the global unitary conjugation but cannot be achieved in general under the local unitary conjugations. Because of this, Zhang et al considered the fidelity between one bipartite quantum state and another one undergoing local unitary dynamics [25]. The problems are related to the geometric measure of entanglement and the distillability problem. 
Besides the above mentioned works, there is also one, where Oszmaniec and Kus [17] estimated the fraction of noncorrelated states within a unitary orbit of a given mixed bipartite state. Based on the obtained result, they have proven that the fraction of noncorrelated states tends to zero exponentially fast with the dimension of the relevant Hilbert space whenever the purity exceeding some critical value. Consequently, a state within a global unitary orbit of a given bipartite state is asymptotically a correlated one. Motivated by this and Page's average entropy formula, in the present paper, we will consider the calculation of average entropy along a global unitary orbit of a given mixed bipartite state. On the one hand, our attempt made here can be seen as a generalization of Page's formula. On the other hand, the obtained result can be viewed as an estimate for the entanglement within a global unitary orbit of a given mixed bipartite state since a random state within a global unitary orbit of a given bipartite state is asymptotically a correlated one. In deriving our main results, we get a lot of by-products which is of independent interest.

The paper is organized as follows. In Sect. 2, we introduce the notion of unitary orbit for quantum states. In calculating the average entropy of a subsystem along a global unitary orbit of a given mixed bipartite state, we need to calculate some integral (Lemma 2.1), which is very important in this paper, over unitary groups. Then, some implications are discussed in Sect. 3. Specifically, we have obtained the following results: (i) For a bipartite quantum system, maximally mixed state can be represented by a uniform probability mixing of tensor products of two marginal states at each point within the global unitary orbit of any given mixed bipartite state; (ii) We use the relative entropy and fidelity as a figure of merit for correlation, and estimate the average correlation along a global unitary orbit from above and below since analytical calculation seems unavailable; (iii) A detailed research is given to the sum of average entropies of two subsystems within a global unitary orbit. We summarize the main contents of this paper in Sect. 4. Finally, we present the detailed proofs of Lemma 2.1, Theorem 2.2, Proposition 3.2, and Theorem 3.4 in Appendix.

\section{Average entropy of a subsystem along a global unitary orbit}

Define unitary orbit $\mathcal{U}_{\rho}$ of a given quantum state $\rho$ on a $d$-dimensional Hilbert space $\mathcal{H}_{d}$ as follows:

$$
\mathcal{U}_{\rho}:=\left\{U \rho U^{\dagger}: U \in \mathrm{U}\left(\mathcal{H}_{d}\right)\right\} .
$$

Choose any $\rho_{A B}^{\prime} \in \mathcal{U}_{\rho_{A B}}$ for a given bipartite state $\rho_{A B}$ and $\rho_{A}^{\prime}=\operatorname{Tr}_{B}\left(\rho_{A B}^{\prime}\right)$. Let $\Phi=\operatorname{Tr}_{B}$, then $\Phi^{*}=\otimes \mathbb{1}_{B}$. Assume that $\Gamma:=\Phi^{*} \Phi$. That is $\Gamma(X)=\operatorname{Tr}_{B}(X) \otimes \mathbb{1}_{B}$.

In what follows, we compute the average entropy along the unitary orbit $\mathcal{U}_{\rho_{A B}}$ of $\rho_{A B}$. By the definition of entropy, $\mathrm{S}\left(\rho_{A}^{\prime}\right)=-\operatorname{Tr}\left(\rho_{A}^{\prime} \ln \rho_{A}^{\prime}\right)$, which can be rewritten as:

$$
\mathrm{S}\left(\rho_{A}^{\prime}\right)=-\operatorname{Tr}\left(\rho_{A B} \ln \left[U^{\dagger} \Gamma\left(U \rho_{A B} U^{\dagger}\right) U\right]\right) .
$$

Note that $-\ln x=\sum_{n=1}^{\infty} \frac{1}{n}(1-x)^{n}$, and it follows that

$$
\begin{aligned}
-\ln \left[U^{\dagger} \Gamma\left(U \rho_{A B} U^{\dagger}\right) U\right] & =\sum_{n=1}^{\infty} \frac{1}{n}\left(\mathbb{1}_{A} \otimes \mathbb{1}_{B}-\left[U^{\dagger} \Gamma\left(U \rho_{A B} U^{\dagger}\right) U\right]\right)^{n} \\
& =\sum_{n=1}^{\infty} \frac{1}{n} U^{\dagger}\left[\Gamma\left(U T U^{\dagger}\right)\right]^{n} U
\end{aligned}
$$


where $T:=\mathbb{1}_{A} \otimes \mathbb{1}_{B} / d_{B}-\rho_{A B}$ with $\operatorname{Tr}(T)=d_{A}-1$. Thus

$$
\int_{\mathrm{U}(d)} \mathrm{S}\left(\rho_{A}^{\prime}\right) \mathrm{d} \mu(U)=\sum_{n=1}^{\infty} \frac{1}{n} \operatorname{Tr}\left(\rho \int U^{\dagger}\left[\Gamma\left(U T U^{+}\right)\right]^{n} U \mathrm{~d} \mu(U)\right),
$$

where $d=d_{A} d_{B}$. Denote

$$
a_{n}:=\operatorname{Tr}\left(\rho \int U^{\dagger}\left[\Gamma\left(U T U^{\dagger}\right)\right]^{n} U \mathrm{~d} \mu(U)\right)
$$

Hence

$$
\ln \left(d_{A}\right) \geqslant \int \mathrm{S}\left(\rho_{A}^{\prime}\right) \mathrm{d} \mu(U)=\sum_{n=1}^{\infty} \frac{a_{n}}{n}
$$

We see from this formula

$$
\lim _{n \rightarrow \infty} a_{n}=0 .
$$

Theoretically, although the specific formula for $a_{n}$ can be obtained $[4,5,22]$, however the specific form of $a_{n}$ will be rather complicated. We can truncate this series (via keeping the first $n$ terms) to obtain an approximation about the average entropy. That is,

$$
\ln \left(d_{A}\right) \geqslant \int \mathrm{S}\left(\rho_{A}^{\prime}\right) \mathrm{d} \mu(U) \geqslant a_{1}+\frac{1}{2} a_{2}+\cdots+\frac{1}{n} a_{n} .
$$

The more larger $n$ is, the more tighter lower bound is. But however, the computing about $a_{n}$ is becoming very complicated when $n \geqslant 3$. As an illustration, we truncate first two terms as an estimate below. More challenging task is to determine the exact value of the above series.

(i). For $n=1$, we have [22]

$$
\int U^{\dagger} \Gamma\left(U T U^{\dagger}\right) U \mathrm{~d} \mu(U)=\frac{d \operatorname{Tr}\left(\Gamma\left(\mathbb{1}_{d}\right)\right)-\operatorname{Tr}(\Gamma)}{d\left(d^{2}-1\right)} \operatorname{Tr}(T) \mathbb{1}_{d}+\frac{d \operatorname{Tr}(\Gamma)-\operatorname{Tr}\left(\Gamma\left(\mathbb{1}_{d}\right)\right)}{d\left(d^{2}-1\right)} T .
$$

Since $\Gamma=\Phi^{*} \Phi$, choose any orthonormal basis $\left\{\left|\varphi_{j}\right\rangle: j=1, \ldots, d_{B}\right\}$ for subsystem space of $B$, it follows that

$$
\Gamma(Z)=\sum_{i, j=1}^{d_{B}} M_{i j} Z M_{i j}^{+},
$$

where $M_{i j}=\mathbb{1}_{A} \otimes\left|\varphi_{i}\right\rangle\left\langle\varphi_{j}\right|=M_{j i}^{\dagger}$, which implies that $\operatorname{Tr}\left(\Gamma\left(\mathbb{1}_{d}\right)\right)=d_{A} d_{B}^{2}, \operatorname{Tr}(\Gamma)=d_{A}^{2} d_{B}$. Thus

$$
\int U^{\dagger} \Gamma\left(U T U^{\dagger}\right) U \mathrm{~d} \mu(U)=\frac{d_{A}-1}{d^{2}-1}\left[\left(1+d d_{B}\right) \mathbb{1}_{A} \otimes \mathbb{1}_{B}-\left(d+d_{B}\right) \rho_{A B}\right] .
$$

Therefore,

$$
a_{1}=\frac{d_{A}-1}{d^{2}-1}\left[\left(1+d d_{B}\right)-\left(d+d_{B}\right) \operatorname{Tr}\left(\rho_{A B}^{2}\right)\right] .
$$

(ii). For $n=2$,

$$
\begin{aligned}
\int U^{+}\left[\Gamma\left(U T U^{\dagger}\right)\right]^{2} U \mathrm{~d} \mu(U) & =\sum_{i, j, k, l=1}^{d_{B}} \int U^{\dagger} M_{i j} U T U^{\dagger}\left(M_{j i} M_{k l}\right) U T U^{\dagger} M_{l k} U \mathrm{~d} \mu(U) \\
& =\sum_{i, j, l=1}^{d_{B}} \int U^{\dagger} M_{i j} U T U^{\dagger} M_{j l} U T U^{\dagger} M_{l i} U \mathrm{~d} \mu(U) .
\end{aligned}
$$

In order to calculate above integral, we will need the following result, described as follows: 
Lemma 2.1. It holds that

$$
\int U A U^{\dagger} B U X U^{\dagger} C U D U^{\dagger} \mathrm{d} \mu(U)=\mu_{1} \cdot \mathbb{1}_{d}+\mu_{2} \cdot B C+\mu_{3} \cdot C B+\mu_{4} \cdot B+\mu_{5} \cdot C,
$$

where the coefficients $\mu_{j}(j=1, \ldots, 5)$ can be found in the Appendix A.

Proof. See Appendix A.

This leads to the following result:

Theorem 2.2. The average von Neumann entropy of a subsystem within the global unitary orbit of a generic mixed bipartite state $\rho_{A B}$, a density matrix on $\mathcal{H}_{d} \equiv \mathcal{H}_{d_{A}} \otimes \mathcal{H}_{d_{B}}$, is bounded from below by a quantity:

$$
\int \mathrm{S}\left(\rho_{A}^{\prime}\right) \mathrm{d} \mu(U) \geqslant a_{1}+\cdots+\frac{a_{n}}{n} \quad(\forall n \in \mathbb{N}),
$$

where

$$
a_{n}=\operatorname{Tr}\left(\rho \int U^{+}\left[\Gamma\left(U T U^{+}\right)\right]^{n} U \mathrm{~d} \mu(U)\right) \geqslant a_{1}^{n} .
$$

In particular, the first two terms can be given specifically:

$$
\begin{aligned}
& a_{1}=\frac{d_{A}-1}{d^{2}-1}\left[\left(1+d d_{B}\right)-\left(d+d_{B}\right) \operatorname{Tr}\left(\rho_{A B}^{2}\right)\right], \\
& a_{2}=\left(f+g \frac{1}{d_{B}}+h \frac{1}{d_{B}^{2}}\right)-\left(g+h \frac{2}{d_{B}}\right) \operatorname{Tr}\left(\rho_{A B}^{2}\right)+h \operatorname{Tr}\left(\rho_{A B}^{3}\right),
\end{aligned}
$$

where $f, g, h$ are given below:

$$
\begin{aligned}
& f=\frac{d_{A}\left(d_{A}^{2}-1\right)\left(d_{B}^{2}-1\right)}{\left(d^{2}-1\right)\left(d^{2}-4\right)}\left(d_{A}+d_{B} \operatorname{Tr}\left(\rho_{A B}^{2}\right)-2\right)+\frac{\left(d^{2}-2 d_{A}^{2}-2\right)\left(d_{B}^{2}-1\right)}{\left(d^{2}-1\right)\left(d^{2}-4\right)}\left(d_{A}-1\right)^{2}, \\
& g==\frac{2 d\left(d_{A}-1\right)\left(d_{A}^{2}-1\right)\left(d_{B}^{2}-1\right)}{\left(d^{2}-1\right)\left(d^{2}-4\right)} \\
& h==\frac{\left(d_{A}^{2}-1\right)\left(d_{A}^{2}-4\right) d_{B}^{2}}{\left(d^{2}-1\right)\left(d^{2}-4\right)} .
\end{aligned}
$$

Moreover,

$$
\int \mathrm{S}\left(\rho_{A}^{\prime}\right) \mathrm{d} \mu(U) \geqslant-\ln \left(1-a_{1}\right)
$$

Proof. See Appendix B.

Remark 2.3. In particular, for $d_{A}=d_{B}=2$, then $d=4$, we have

$$
a_{1}=\frac{3}{5}-\frac{2}{5} \operatorname{Tr}\left(\rho_{A B}^{2}\right), \quad a_{2}=\frac{3}{10}-\frac{1}{5} \operatorname{Tr}\left(\rho_{A B}^{2}\right) .
$$

This implies that

$$
\ln 2 \geqslant \int \mathrm{S}\left(\rho_{A}^{\prime}\right) \mathrm{d} \mu(U) \geqslant \max \left\{\frac{9}{10}-\frac{3}{5} \operatorname{Tr}\left(\rho_{A B}^{2}\right), \ln 5-\ln \left(2+2 \operatorname{Tr}\left(\rho_{A B}^{2}\right)\right)\right\} .
$$

It is left open that the explicit computing of the following integral for a given mixed bipartite state $\rho_{A B}$ :

$$
\int \ln \left(U^{\dagger} \Gamma\left(U \rho_{A B} U^{\dagger}\right) U\right) \mathrm{d} \mu(U)
$$


Remark 2.4. We have already known that for any super-operator $\Xi$ over $\mathcal{H}_{d}[22]$,

$$
\int_{\mathrm{U}(d)} \mathrm{d} \mu(U) U^{\dagger} \Xi\left(U X U^{\dagger}\right) U=\frac{d \operatorname{Tr}\left(\Xi\left(\mathbb{1}_{d}\right)\right)-\operatorname{Tr}(\Xi)}{d\left(d^{2}-1\right)} \operatorname{Tr}(X) \mathbb{1}_{d}+\frac{d \operatorname{Tr}(\Xi)-\operatorname{Tr}\left(\Xi\left(\mathbb{1}_{d}\right)\right)}{d\left(d^{2}-1\right)} X .
$$

Now let $d=d_{A} d_{B}$ and $\mathcal{H}_{d}=\mathcal{H}_{A} \otimes \mathcal{H}_{B}$ with $\operatorname{dim}\left(\mathcal{H}_{A}\right)=d_{A}$ and $\operatorname{dim}\left(\mathcal{H}_{B}\right)=d_{B}$. Assume that $X=\rho_{A B}$, a density matrix on $\mathcal{H}_{A} \otimes \mathcal{H}_{B}$. Fixing an orthonormal basis $\left\{\left|\psi_{B, j}\right\rangle: j=1, \ldots, d_{B}\right\}$ for $\mathcal{H}_{B}$. Suppose that $\Gamma(X)=\operatorname{Tr}_{B}(X) \otimes \mathbb{1}_{B}$. Then it can be rewritten as:

$$
\Gamma(X)=\sum_{i, j=1}^{d_{B}}\left(\mathbb{1}_{A} \otimes\left|\psi_{B, i}\right\rangle\left\langle\psi_{B, j}\right|\right) X\left(\mathbb{1}_{A} \otimes\left|\psi_{B, j}\right\rangle\left\langle\psi_{B, i}\right|\right) .
$$

Clearly $\Gamma\left(\mathbb{1}_{A} \otimes \mathbb{1}_{B}\right)=d_{B} \mathbb{1}_{A} \otimes \mathbb{1}_{B}$, implying that

$$
\operatorname{Tr}\left(\Gamma\left(\mathbb{1}_{A} \otimes \mathbb{1}_{B}\right)\right)=d_{A} d_{B}^{2} \text { and } \operatorname{Tr}(\Gamma)=\sum_{i, j=1}^{d_{B}}\left(d_{A} \delta_{i j}\right)^{2}=d_{A}^{2} d_{B} .
$$

From the above discussion, we see that

$$
\int_{\mathrm{U}(d)} \mathrm{d} \mu(U) U^{\dagger} \Gamma\left(U \rho_{A B} U^{\dagger}\right) U=\frac{d d_{B}-d_{A}}{d^{2}-1} \mathbb{1}_{A} \otimes \mathbb{1}_{B}+\frac{d d_{A}-d_{B}}{d^{2}-1} \rho_{A B} .
$$

Denote $\rho_{A B}^{\prime}=U \rho_{A B} U^{+}$and $\rho_{A}^{\prime}=\operatorname{Tr}_{B}\left(\rho_{A B}^{\prime}\right)$. Then

$$
\begin{aligned}
\operatorname{Tr}\left(\left(\rho_{A}^{\prime}\right)^{2}\right) & =\operatorname{Tr}\left(\left(\rho_{A}^{\prime} \otimes \mathbb{1}_{B}\right) \rho_{A B}^{\prime}\right)=\operatorname{Tr}\left(\Gamma\left(\rho_{A B}^{\prime}\right) \rho_{A B}^{\prime}\right) \\
& =\operatorname{Tr}\left(U^{\dagger} \Gamma\left(U \rho_{A B} U^{\dagger}\right) U \rho_{A B}\right) .
\end{aligned}
$$

Therefore

$$
\left\langle\operatorname{Tr}\left(\left(\rho_{A}^{\prime}\right)^{2}\right)\right\rangle:=\int \mathrm{d} \mu(U) \operatorname{Tr}\left(\left(\rho_{A}^{\prime}\right)^{2}\right)=\operatorname{Tr}\left(\int \mathrm{d} \mu(U) U^{\dagger} \Gamma\left(U \rho_{A B} U^{\dagger}\right) U \rho_{A B}\right) .
$$

That is,

$$
\left\langle\operatorname{Tr}\left(\left(\rho_{A}^{\prime}\right)^{2}\right)\right\rangle=\frac{d d_{B}-d_{A}}{d^{2}-1}+\frac{d d_{A}-d_{B}}{d^{2}-1} \operatorname{Tr}\left(\rho_{A B}^{2}\right) .
$$

If we use the notion of linear entropy $S_{L}(\rho):=1-\operatorname{Tr}\left(\rho^{2}\right)$, then we have seen from Eq. (2.19) that the average linear entropy is given precisely by

$$
\int \mathrm{S}_{L}\left(\rho_{A}^{\prime}\right) \mathrm{d} \mu(U)=\frac{\left(d_{A}-1\right)\left(d_{B}-1\right)}{d+1}+\frac{d d_{A}-d_{B}}{d^{2}-1} \mathrm{~S}_{L}\left(\rho_{A B}\right) .
$$

We see from this that

$$
\int \mathrm{S}_{L}\left(\rho_{B}^{\prime}\right) \mathrm{d} \mu(U)=\frac{\left(d_{A}-1\right)\left(d_{B}-1\right)}{d+1}+\frac{d d_{B}-d_{A}}{d^{2}-1} \mathrm{~S}_{L}\left(\rho_{A B}\right)
$$

Moreover for a mixed state $\rho_{A B}, \int \mathrm{S}_{L}\left(\rho_{A}^{\prime}\right) \mathrm{d} \mu(U)=\int \mathrm{S}_{L}\left(\rho_{B}^{\prime}\right) \mathrm{d} \mu(U)$ if and only if $d_{A}=d_{B}$. We also see that

$$
\int\left(\mathrm{S}_{L}\left(\rho_{A}^{\prime}\right)+\mathrm{S}_{L}\left(\rho_{B}^{\prime}\right)\right) \mathrm{d} \mu(U)=2 \frac{\left(d_{A}-1\right)\left(d_{B}-1\right)}{d+1}+\frac{d_{A}+d_{B}}{d+1} \mathrm{~S}_{L}\left(\rho_{A B}\right) .
$$

In particular, if $d_{A}=d_{B}=2$, then

$$
\int \mathrm{S}_{L}\left(\rho_{A}^{\prime}\right) \mathrm{d} \mu(U)=\int \mathrm{S}_{L}\left(\rho_{B}^{\prime}\right) \mathrm{d} \mu(U)=\frac{1}{5}+\frac{2}{5} \mathrm{~S}_{L}\left(\rho_{A B}\right) .
$$




\section{Some implications}

Some consequences of Lemma 2.1 can be presented as follows: Let $\Phi=\operatorname{Tr}_{B}$ and $\Psi=\operatorname{Tr}_{A}$. Then $\Phi^{*}=\otimes \mathbb{1}_{B}$ and $\Psi^{*}=\mathbb{1}_{A} \otimes$. Thus for $\rho_{A B}^{\prime}=U \rho_{A B} U^{\dagger}$,

$$
\begin{aligned}
& \rho_{A}^{\prime} \otimes \rho_{B}^{\prime}=\Phi^{*} \Phi\left(U \rho_{A B} U^{\dagger}\right) \Psi^{*} \Psi\left(U \rho_{A B} U^{\dagger}\right) \\
& =\sum_{i, j=1}^{d_{B}} \sum_{\mu, v=1}^{d_{A}}\left(\mathbb{1}_{A} \otimes|i\rangle\langle j|\right) U \rho_{A B} U^{\dagger}(|\mu\rangle\langle v|\otimes| j\rangle\langle i|) U \rho_{A B} U^{\dagger}\left(|v\rangle\langle\mu| \otimes \mathbb{1}_{B}\right),
\end{aligned}
$$

and it follows that

$$
\int\left(\rho_{A}^{\prime} \otimes \rho_{B}^{\prime}\right) \mathrm{d} \mu(U)=\sum_{i, j=1}^{d_{B}} \sum_{\mu, v=1}^{d_{A}}\left(\mathbb{1}_{A} \otimes|i\rangle\langle j|\right)\left(\int U \rho_{A B} U^{\dagger}(|\mu\rangle\langle v|\otimes| j\rangle\langle i|) U \rho_{A B} U^{\dagger} d U\right)\left(|v\rangle\langle\mu| \otimes \mathbb{1}_{B}\right),
$$

leading to the following identity

$$
\int\left(\rho_{A}^{\prime} \otimes \rho_{B}^{\prime}\right) \mathrm{d} \mu(U)=\frac{\mathbb{1}_{A}}{d_{A}} \otimes \frac{\mathbb{1}_{B}}{d_{B}} .
$$

This amounts to say that

Proposition 3.1. For a bipartite quantum system, maximally mixed state can be represented by a uniform probability mixing of tensor products of two marginal states at each point within the global unitary orbit of any mixed bipartite state.

Besides, we also get that

$$
\begin{aligned}
& \int U^{\dagger}\left(\rho_{A}^{\prime} \otimes \rho_{B}^{\prime}\right) U \mathrm{~d} \mu(U) \\
& =\sum_{i, j=1}^{d_{B}} \sum_{\mu, v=1}^{d_{A}} \int U^{\dagger}\left(\mathbb{1}_{A} \otimes|i\rangle\langle j|\right) U \rho_{A B} U^{\dagger}(|\mu\rangle\langle v|\otimes| j\rangle\langle i|) U \rho_{A B} U^{\dagger}\left(|v\rangle\langle\mu| \otimes \mathbb{1}_{B}\right) U \mathrm{~d} \mu(U),
\end{aligned}
$$

leading to the following identity

$$
\int U^{\dagger}\left(\rho_{A}^{\prime} \otimes \rho_{B}^{\prime}\right) U \mathrm{~d} \mu(U)=c_{0} \cdot \mathbb{1}_{d}+c_{1} \cdot \rho_{A B}+c_{2} \cdot \rho_{A B}^{2},
$$

where

$$
\begin{aligned}
& c_{0}=\frac{\left(d_{A}^{2}-1\right)\left(d_{B}^{2}-1\right)}{\left(d^{2}-1\right)\left(d^{2}-4\right)}\left(d-2 \operatorname{Tr}\left(\rho_{A B}^{2}\right)\right), \\
& c_{1}=\frac{d^{2}\left(d_{A}^{2}+d_{B}^{2}-6\right)+4}{\left(d^{2}-1\right)\left(d^{2}-4\right)}, \\
& c_{2}=\frac{2 d\left(d_{A}^{2}-1\right)\left(d_{B}^{2}-1\right)}{\left(d^{2}-1\right)\left(d^{2}-4\right)} .
\end{aligned}
$$

The total correlation attained between the subsystems of a bipartite quantum system is constrained if the bipartite system undergoes global unitary evolutions. The authors of Ref [11, 12] investigated related problems from the field of quantum thermodynamics. Since knowing the maximal possible variation in 
correlations is useful, it raises the optimization problem, where a search of the maximal and minimal correlated states on a unitary orbit is needed. This is completely solved for two-qubit systems.

In the following, we make an attempt to calculate the average quantum mutual information (QMI) along a unitary orbit of a generic mixed bipartite state. We provide some analytical upper and/or lower bounds for the average QMI and quantum fidelity along a unitary orbit, although we cannot obtain precise formulas for them.

Proposition 3.2. A lower bound for the maximal correlation (defined by relative entropy) within the global unitary orbit of a mixed bipartite state $\rho_{A B}$ is given as follows

$$
\int I(A: B)_{\rho^{\prime}} \mathrm{d} \mu(U) \geqslant \mathrm{S}\left(\rho_{A B} \| c_{0} \cdot \mathbb{1}_{d}+c_{1} \cdot \rho_{A B}+c_{2} \cdot \rho_{A B}^{2}\right),
$$

where $I(A: B)_{\rho}:=\mathrm{S}\left(\rho_{A B} \| \rho_{A} \otimes \rho_{B}\right)$, where $\mathrm{S}(\rho \| \sigma):=\operatorname{Tr}(\rho(\ln \rho-\ln \sigma))$ is the relative entropy. Similarly, the lower and upper bounds for the average fidelity within the global unitary orbit of a mixed bipartite state $\rho_{A B}$ are given as follows

$$
c_{0}+c_{1} \operatorname{Tr}\left(\rho_{A B}^{2}\right)+c_{2} \operatorname{Tr}\left(\rho_{A B}^{3}\right) \leqslant \int \mathrm{F}\left(\rho_{A B}^{\prime}, \rho_{A}^{\prime} \otimes \rho_{B}^{\prime}\right) \mathrm{d} \mu(U) \leqslant \mathrm{F}\left(\rho_{A B}, c_{0} \cdot \mathbb{1}_{d}+c_{1} \cdot \rho_{A B}+c_{2} \cdot \rho_{A B}^{2}\right) .
$$

where $\mathrm{F}(\rho, \sigma):=\operatorname{Tr}(\sqrt{\sqrt{\rho} \sigma \sqrt{\rho}})$ is the fidelity between two states $\rho$ and $\sigma$.

\section{Proof. See Appendix C.}

Remark 3.3. Recently, in [9] the authors introduced a general measure of correlations for two-qubit states based on the classical mutual information between local observables. They focus on (classical) correlations between sets of local observables instead of the quantum vs classical distinction. In this perspective, quantum states can be characterized as a whole by the average amount of (classical) correlations between all pairs of local observables. Under some restrictions, the authors calculated the average mutual information, whose value depends on the state purity, and the symmetry of the correlations distribution.

For the two-qubit case, we apply the spectral decomposition $\rho_{A B}=U \Lambda U^{\dagger}$ with $\Lambda=\operatorname{diag}\left(\lambda_{1}, \lambda_{2}, \lambda_{3}, \lambda_{4}\right)$, where

$$
\left(\lambda_{1}, \lambda_{2}, \lambda_{3}, \lambda_{4}\right)^{\top} \in \Delta_{3}=\left\{\left(p_{1}, p_{2}, p_{3}, p_{4}\right)^{\top} \in \mathbb{R}^{4}: p_{j} \geqslant 0(\forall j=1,2,3,4), \sum_{j=1}^{4} p_{j}=1\right\} .
$$

Due to the invariance under unitary conjugation, we have

$$
\mathrm{F}\left(\Lambda, c_{0}+c_{1} \Lambda+c_{2} \Lambda^{2}\right)=\operatorname{Tr}\left(\sqrt{\sqrt{\Lambda}\left(c_{0}+c_{1} \Lambda+c_{2} \Lambda^{2}\right) \sqrt{\Lambda}}\right)=\sum_{j=1}^{4}\left(c_{0} \lambda_{j}+c_{1} \lambda_{j}^{2}+c_{2} \lambda_{j}^{3}\right)^{\frac{1}{2}} .
$$

The lower bound in (3.9) becomes

$$
c_{0}+c_{1} \operatorname{Tr}\left(\Lambda^{2}\right)+c_{2} \operatorname{Tr}\left(\Lambda^{3}\right)=c_{0}+\sum_{j=1}^{4}\left(c_{1} \lambda_{j}^{2}+c_{2} \lambda_{j}^{3}\right) .
$$

We numerically check the bounds in (3.9) by choosing 10000 random points in the probability simplex $\Delta_{3}$ and drawing the 3D scatter plot of $\left(\mathcal{P}(\Lambda), c_{0}+c_{1} \operatorname{Tr}\left(\Lambda^{2}\right)+c_{2} \operatorname{Tr}\left(\Lambda^{3}\right), \mathrm{F}\left(\Lambda, c_{0}+c_{1} \Lambda+c_{2} \Lambda^{2}\right)\right)$. For 
clarity, its three-view drawings are also given by using the corresponding 2D scatter plots: $\mathcal{P}(\Lambda)$ vs. $\mathrm{F}\left(\Lambda, c_{0}+c_{1} \Lambda+c_{2} \Lambda^{2}\right)$ in Figure $1(\mathrm{~b}) ; c_{0}+c_{1} \operatorname{Tr}\left(\Lambda^{2}\right)+c_{2} \operatorname{Tr}\left(\Lambda^{3}\right)$ vs. $\mathrm{F}\left(\Lambda, c_{0}+c_{1} \Lambda+c_{2} \Lambda^{2}\right)$ in Figure 1(c); and $\mathcal{P}(\Lambda)$ vs. $c_{0}+c_{1} \operatorname{Tr}\left(\Lambda^{2}\right)+c_{2} \operatorname{Tr}\left(\Lambda^{3}\right)$ in Figure $1(\mathrm{~d})$. We also notice that the maximum of $c_{0}+c_{1} \operatorname{Tr}\left(\Lambda^{2}\right)+c_{2} \operatorname{Tr}\left(\Lambda^{3}\right)$ is even less than the minimum of $\mathrm{F}\left(\Lambda, c_{0}+c_{1} \Lambda+c_{2} \Lambda^{2}\right)$.
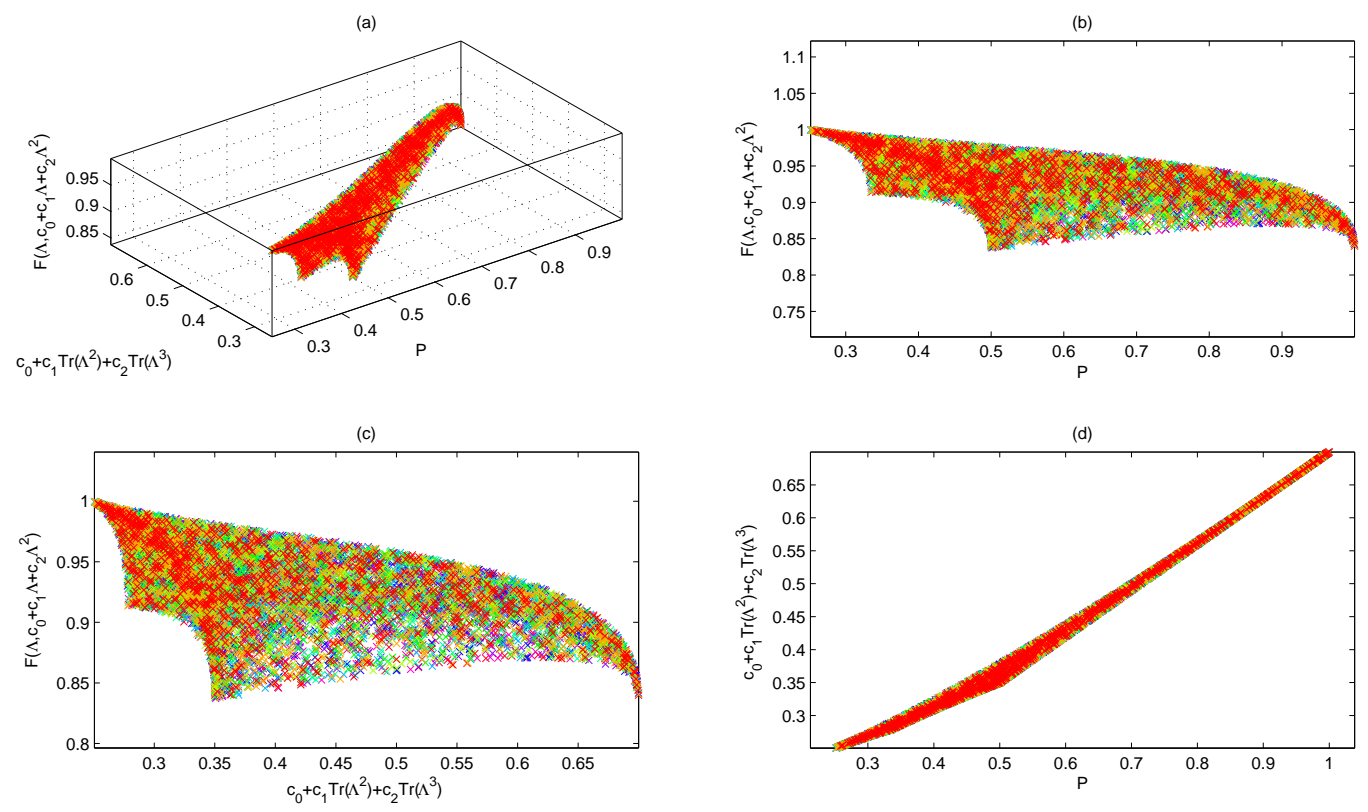

Figure 1: Two-qubit case. (a) 3D scatter plot of $\left(\mathcal{P}(\Lambda), c_{0}+c_{1} \operatorname{Tr}\left(\Lambda^{2}\right)+c_{2} \operatorname{Tr}\left(\Lambda^{3}\right), \mathrm{F}\left(\Lambda, c_{0}+c_{1} \Lambda+c_{2} \Lambda^{2}\right)\right.$ ). $2 \mathrm{D}$ scatter plots: (b) $\mathcal{P}(\Lambda)$ vs. $\mathrm{F}\left(\Lambda, c_{0}+c_{1} \Lambda+c_{2} \Lambda^{2}\right)$; (c) $c_{0}+c_{1} \operatorname{Tr}\left(\Lambda^{2}\right)+c_{2} \operatorname{Tr}\left(\Lambda^{3}\right)$ vs. $\mathrm{F}\left(\Lambda, c_{0}+c_{1} \Lambda+\right.$ $\left.c_{2} \Lambda^{2}\right) ;\left(\right.$ d) $\mathcal{P}(\Lambda)$ vs. $c_{0}+c_{1} \operatorname{Tr}\left(\Lambda^{2}\right)+c_{2} \operatorname{Tr}\left(\Lambda^{3}\right)$.

In fact, not only the value of the maximal QMI is obtained, but also the maximum QMI state $\rho_{\max }$ is derived in [11, 12]. Specifically, $\rho_{A B}=U \Lambda U^{+}$for $d_{A}=d_{B}$, where $\Lambda=\left\{\lambda_{j}: j=1, \ldots, d=d_{A} d_{B}\right\}$ is the spectrum of $\rho_{A B}$,

$$
\max _{\rho_{A B}^{\prime} \in \mathcal{U}_{\rho_{A B}}} I(A: B)_{\rho^{\prime}}=I(A: B)_{\rho_{\max }}=\ln (d)-\mathrm{S}(\Lambda),
$$

where

$$
\rho_{\max }=\sum_{j=1}^{d} \lambda_{j}\left|\Omega_{j}\right\rangle\left\langle\Omega_{j}\right|
$$

for any generalized Bell-state basis $\left\{\left|\Omega_{j}\right\rangle\right\}$. Unlike the maximum QMI case, finding $\rho_{\min }$ on the unitary orbit is more difficult than finding $\rho_{\max }$ in general. Since QMI varies on a unitary orbit completely depends on the sum of two marginal entries, we need to figure out the spectra of two reduced states of all the states in the unitary orbit $\mathcal{U}_{\rho}$. The literature indicates that calculating this set of compatible reduced states with a given spectrum of a global bipartite seems unforeseeable. This is well-known "quantum marginal problem", which is fully solved for two-qubit case [1] and theoretically for two-qubit case [13]. Specifically, 
for two-qubit case, the solution of quantum marginal problem is given by the following: Mixed two-qubit state $\rho_{A B}$ with spectrum $\Lambda=\left\{\lambda_{1} \geqslant \lambda_{2} \geqslant \lambda_{3} \geqslant \lambda_{4} \geqslant 0\right\}$ and the marginal states $\rho_{A}$ and $\rho_{B}$ exist if and only if minimal eigenvalues $\lambda_{\min }^{A}$ and $\lambda_{\min }^{B}$ of the marginal states satisfy the inequalities [1]:

$$
\left\{\begin{array}{l}
\min \left(\lambda_{\min }^{A}, \lambda_{\min }^{B}\right) \geqslant \lambda_{3}+\lambda_{4} \\
\lambda_{\min }^{A}+\lambda_{\min }^{B} \geqslant \lambda_{2}+\lambda_{3}+2 \lambda_{4} \\
\left|\lambda_{\min }^{A}-\lambda_{\min }^{B}\right| \leqslant \min \left(\lambda_{1}-\lambda_{3}, \lambda_{2}-\lambda_{4}\right) .
\end{array}\right.
$$

With the help of this result, the value of the minimal QMI on a unitary orbit is derived for two-qubit case [11, 12]:

$$
I(A: B)_{\rho_{\min }}=h\left(\lambda_{1}+\lambda_{2}\right)+h\left(\lambda_{1}+\lambda_{3}\right)-\mathrm{S}(\Lambda),
$$

where $\lambda_{1} \geqslant \lambda_{2} \geqslant \lambda_{3} \geqslant \lambda_{4} \geqslant 0$ and $h(p):=p \ln p+(1-p) \ln (1-p)$ is the binary entropy function defined for $p \in[0,1]$, moreover two-qubit state $\rho_{\min }$ is given by

$$
\rho_{\min }=\sum_{i, j=1}^{0} \lambda_{i j}|i j\rangle\langle i j| .
$$

Here $\lambda_{i j}$ is a re-indexing of $\lambda_{k}\left(\lambda_{00}=\lambda_{1}, \lambda_{01}=\lambda_{2}, \ldots\right)$ and $\{|i\rangle\},\{|j\rangle\}$ are qubit basis states for subsystems $A$ and $B$.

By Eq. (3.8) and the concavity of von Neumann entropy, we can draw the following conclusion:

Theorem 3.4. For any generic mixed bipartite state $\rho_{A B}$, denoting

$$
\left\langle S_{A}+S_{B}\right\rangle:=\int \mathrm{S}\left(\rho_{A}^{\prime}\right) \mathrm{d} \mu(U)+\int \mathrm{S}\left(\rho_{B}^{\prime}\right) \mathrm{d} \mu(U)
$$

we have that

$$
\mathrm{S}\left(\rho_{A B}\right)+\mathrm{S}\left(\rho_{A B} \| c_{0} \cdot \mathbb{1}_{d}+c_{1} \cdot \rho_{A B}+c_{2} \cdot \rho_{A B}^{2}\right) \leqslant\left\langle S_{A}+S_{B}\right\rangle \leqslant \mathrm{S}\left(c_{0} \cdot \mathbb{1}_{d}+c_{1} \cdot \rho_{A B}+c_{2} \cdot \rho_{A B}^{2}\right),
$$

where $c_{j}(j=0,1,2)$ are given by (3.5), (3.6), and (3.7), respectively. Furthermore,

$$
\mathrm{S}\left(\rho_{A B} \| c_{0} \cdot \mathbb{1}_{d}+c_{1} \cdot \rho_{A B}+c_{2} \cdot \rho_{A B}^{2}\right) \leqslant \int I(A: B)_{\rho^{\prime}} \mathrm{d} \mu(U) \leqslant \mathrm{S}\left(c_{0} \cdot \mathbb{1}_{d}+c_{1} \cdot \rho_{A B}+c_{2} \cdot \rho_{A B}^{2}\right)-\mathrm{S}\left(\rho_{A B}\right) .
$$

In particular, we get a universal entropy inequality:

$$
\mathrm{S}\left(\rho_{A B} \| c_{0} \cdot \mathbb{1}_{d}+c_{1} \cdot \rho_{A B}+c_{2} \cdot \rho_{A B}^{2}\right) \leqslant \mathrm{S}\left(c_{0} \cdot \mathbb{1}_{d}+c_{1} \cdot \rho_{A B}+c_{2} \cdot \rho_{A B}^{2}\right)-\mathrm{S}\left(\rho_{A B}\right) .
$$

Moreover, $\rho_{A B}=c_{0} \cdot \mathbb{1}_{d}+c_{1} \cdot \rho_{A B}+c_{2} \cdot \rho_{A B}^{2}$ if and only if $\rho_{A B}$ is maximally mixed state, i.e., $\rho_{A B}=\frac{\mathbb{1}_{A}}{d_{A}} \otimes \frac{\mathbb{1}_{B}}{d_{B}}$.

\section{Proof. See Appendix D.}

This result tells us whenever the state $\rho_{A B}$ is not maximally mixed state, we can always find correlated states on a global unitary orbit of this state. Indeed, all quantum states are unitarily connected to classical states, one-way or fully classical [16]. 
In particular, for the balanced bipartite system, i.e., $d_{A}=d_{B}$, we have, for $d=d_{A} d_{B}$,

$$
\int \mathrm{S}\left(\rho_{A}^{\prime}\right) \mathrm{d} \mu(U)+\int \mathrm{S}\left(\rho_{B}^{\prime}\right) \mathrm{d} \mu(U) \leqslant \max _{U}\left(\mathrm{~S}\left(\rho_{A}^{\prime}\right)+\mathrm{S}\left(\rho_{B}^{\prime}\right)\right)=\max _{U} I(A: B)_{\rho^{\prime}}+\mathrm{S}\left(\rho_{A B}\right)=\ln (d),
$$

where we used the fact that $\max _{U} I(A: B)_{\rho^{\prime}}=\ln (d)-\mathrm{S}\left(\rho_{A B}\right)$ [11]. Apparently, this upper bound is true for $d_{A} \neq d_{B}$, and moreover it is trivially since $S\left(\rho_{X}^{\prime}\right) \leqslant \ln \left(d_{X}\right)$, where $X=A, B$. Thus the following inequality is always true

$$
\int \mathrm{S}\left(\rho_{A}^{\prime}\right) \mathrm{d} \mu(U)+\int \mathrm{S}\left(\rho_{B}^{\prime}\right) \mathrm{d} \mu(U) \leqslant \ln (d) .
$$

In view of this, we get a tighter upper bound for the sum of the average entropy of two subsystems:

$$
\int \mathrm{S}\left(\rho_{A}^{\prime}\right) \mathrm{d} \mu(U)+\int \mathrm{S}\left(\rho_{B}^{\prime}\right) \mathrm{d} \mu(U) \leqslant \mathrm{S}\left(c_{0} \cdot \mathbb{1}_{d}+c_{1} \cdot \rho_{A B}+c_{2} \cdot \rho_{A B}^{2}\right) \leqslant \ln (d) .
$$

We will check the difference between the upper bound $S\left(c_{0} \cdot \mathbb{1}_{d}+c_{1} \cdot \rho_{A B}+c_{2} \cdot \rho_{A B}^{2}\right)$ and the lower bound $\mathrm{S}\left(\rho_{A B}\right)+\mathrm{S}\left(\rho_{A B} \| c_{0} \cdot \mathbb{1}_{d}+c_{1} \cdot \rho_{A B}+c_{2} \cdot \rho_{A B}^{2}\right)$ since we want to identify the range of the sum of two average entropy. Let

$$
\mathcal{F}\left(\rho_{A B}\right):=\mathrm{S}\left(c_{0} \cdot \mathbb{1}_{d}+c_{1} \cdot \rho_{A B}+c_{2} \cdot \rho_{A B}^{2}\right)-\mathrm{S}\left(\rho_{A B}\right)-\mathrm{S}\left(\rho_{A B} \| c_{0} \cdot \mathbb{1}_{d}+c_{1} \cdot \rho_{A B}+c_{2} \cdot \rho_{A B}^{2}\right)
$$

and $\mathcal{P}=\operatorname{Tr}\left(\rho_{A B}^{2}\right)$, which lies in $\left[d^{-1}, 1\right]$ for $d=d_{A} d_{B}$. Apparently, $\mathcal{F} \geqslant 0$ over the whole set of states.

$$
\text { If } d_{A}=d_{B}=2 \text {, then }
$$

$$
c_{0}=\frac{2-\mathcal{P}}{10}, \quad c_{1}=\frac{1}{5}, \quad c_{2}=\frac{2}{5} .
$$

Thus in the two-qubit case, we have

$$
\int U^{\dagger}\left(\rho_{A}^{\prime} \otimes \rho_{B}^{\prime}\right) U \mathrm{~d} \mu(U)=\frac{2-\mathcal{P}}{10} \cdot \mathbb{1}_{4}+\frac{1}{5} \cdot \rho_{A B}+\frac{2}{5} \cdot \rho_{A B}^{2} .
$$

Note that $S\left(\rho_{A B}\right)+\mathrm{S}\left(\rho_{A B} \| c_{0} \cdot \mathbb{1}_{d}+c_{1} \cdot \rho_{A B}+c_{2} \cdot \rho_{A B}^{2}\right)=-\operatorname{Tr}\left(\rho_{A B} \ln \left[c_{0} \cdot \mathbb{1}_{d}+c_{1} \cdot \rho_{A B}+c_{2} \cdot \rho_{A B}^{2}\right]\right)$. Now $\mathcal{F}$ is reduced to the following form:

$$
\begin{aligned}
\mathcal{F}\left(\rho_{A B}\right)= & S\left(\frac{2-\mathcal{P}}{10} \cdot \mathbb{1}_{4}+\frac{1}{5} \cdot \rho_{A B}+\frac{2}{5} \cdot \rho_{A B}^{2}\right) \\
& +\operatorname{Tr}\left(\rho_{A B} \ln \left[\frac{2-\mathcal{P}}{10} \cdot \mathbb{1}_{d}+\frac{1}{5} \cdot \rho_{A B}+\frac{2}{5} \cdot \rho_{A B}^{2}\right]\right) .
\end{aligned}
$$

Since $\mathcal{F}$ is invariant under unitary conjugation, it follows, via the spectral decomposition $\rho=U \Lambda U^{\dagger}$ of $\rho_{A B}$, where $\Lambda=\operatorname{diag}\left(\lambda_{1}, \lambda_{2}, \lambda_{3}, \lambda_{4}\right)$, that

$$
\begin{aligned}
\mathcal{F}(\Lambda)= & S\left(\frac{2-\mathcal{P}}{10} \cdot \mathbb{1}_{4}+\frac{1}{5} \cdot \Lambda+\frac{2}{5} \cdot \Lambda^{2}\right) \\
& +\operatorname{Tr}\left(\Lambda \ln \left[\frac{2-\mathcal{P}}{10} \cdot \mathbb{1}_{4}+\frac{1}{5} \cdot \Lambda+\frac{2}{5} \cdot \Lambda^{2}\right]\right) .
\end{aligned}
$$

We see that $\mathcal{F}$ is a symmetric function defined over the probability simplex

$$
\Delta_{3}=\left\{\left(p_{1}, p_{2}, p_{3}, p_{4}\right)^{\top} \in \mathbb{R}^{4}: p_{j} \geqslant 0(\forall j=1,2,3,4), \sum_{j=1}^{4} p_{j}=1\right\} .
$$


Next we make numerical analysis of this function defined over the probability simplex.

$$
\begin{aligned}
\mathcal{F}\left(\lambda_{1}, \lambda_{2}, \lambda_{3}, \lambda_{4}\right)= & \sum_{j=1}^{4} \lambda_{j} \ln \left(\frac{2-\mathcal{P}}{10}+\frac{1}{5} \lambda_{j}+\frac{2}{5} \lambda_{j}^{2}\right) \\
& -\left(\frac{2-\mathcal{P}}{10}+\frac{1}{5} \lambda_{j}+\frac{2}{5} \lambda_{j}^{2}\right) \ln \left(\frac{2-\mathcal{P}}{10}+\frac{1}{5} \lambda_{j}+\frac{2}{5} \lambda_{j}^{2}\right),
\end{aligned}
$$

where $\left(\lambda_{1}, \lambda_{2}, \lambda_{3}, \lambda_{4}\right)^{\top} \in \Delta_{3}$ and $\mathcal{P} \equiv \mathcal{P}(\Lambda):=\operatorname{Tr}\left(\Lambda^{2}\right)=\sum_{j=1}^{4} \lambda_{j}^{2}$.

Choose random point $\Lambda$ in the probability simplex $\Delta_{3}$, and then draw the 3-dimensional figure about the 3-tuples $(x, y, z)$ where $x=\mathcal{P}(\Lambda), y=-\operatorname{Tr}\left(\Lambda \ln \left[\frac{2-\mathcal{P}(\Lambda)}{10} \cdot \mathbb{1}_{4}+\frac{1}{5} \cdot \Lambda+\frac{2}{5} \cdot \Lambda^{2}\right]\right), z=\mathrm{S}\left(c_{0}+c_{1} \Lambda+\right.$ $\left.c_{2} \Lambda^{2}\right)$. To illustrate the relationship among $\mathcal{P}(\Lambda), \mathrm{S}\left(\rho_{A B}\right)+\mathrm{S}\left(\rho_{A B} \| c_{0} \cdot \mathbb{1}_{d}+c_{1} \cdot \rho_{A B}+c_{2} \cdot \rho_{A B}^{2}\right)$ and $\mathrm{S}\left(c_{0}+\right.$ $\left.c_{1} \Lambda+c_{2} \Lambda^{2}\right)$, we show the 3D scatter plot in Figure 2(a), where there are 5000 random points used in total. To make things more clear, we also demonstrate $2 \mathrm{D}$ scatter plots: $\mathrm{S}\left(\rho_{A B}\right)+\mathrm{S}\left(\rho_{A B} \| c_{0} \cdot \mathbb{1}_{d}+c_{1} \cdot \rho_{A B}+c_{2} \cdot \rho_{A B}^{2}\right)$ vs. $S\left(c_{0}+c_{1} \Lambda+c_{2} \Lambda^{2}\right)$ in Figure $2(b), \mathcal{P}$ vs. $S\left(c_{0}+c_{1} \Lambda+c_{2} \Lambda^{2}\right)$ in Figure $2(\mathrm{c})$, and $\mathcal{P}$ vs. $\mathcal{F}$ in Figure $2(\mathrm{~d})$. Note that for the case where $\lambda_{j}=\frac{1}{4}(j=1,2,3,4)$ we have $\mathrm{S}(\Lambda)=\mathrm{S}\left(c_{0}+c_{1} \Lambda+c_{2} \Lambda^{2}\right)=$ $\ln 4=1.39$ and $\mathcal{F}=0$; while the case with one $\lambda_{j}$ equaling to 1 and the other three being zeros yields $\mathrm{S}(\Lambda)=0, \mathrm{~S}\left(c_{0}+c_{1} \Lambda+c_{2} \Lambda^{2}\right)=-\frac{3}{10} \ln \frac{1}{10}-\frac{7}{10} \ln \frac{7}{10}=0.94, \operatorname{Tr}\left(\Lambda \ln \left(c_{0}+c_{1} \Lambda+c_{2} \Lambda^{2}\right)\right)=\ln \frac{7}{10}$ and $\mathcal{F}=-\frac{3}{10} \ln \frac{1}{10}-\frac{3}{5} \ln \frac{7}{10}=0.58$. In these plots, $\mathcal{P} \in\left[\frac{1}{4}, 1\right], \mathrm{S}(\Lambda) \in[0, \ln 4], \mathrm{S}\left(c_{0}+c_{1} \Lambda+c_{2} \Lambda^{2}\right) \in[0.94, \ln 4]$ and $\mathcal{F} \in[0,0.58]$.
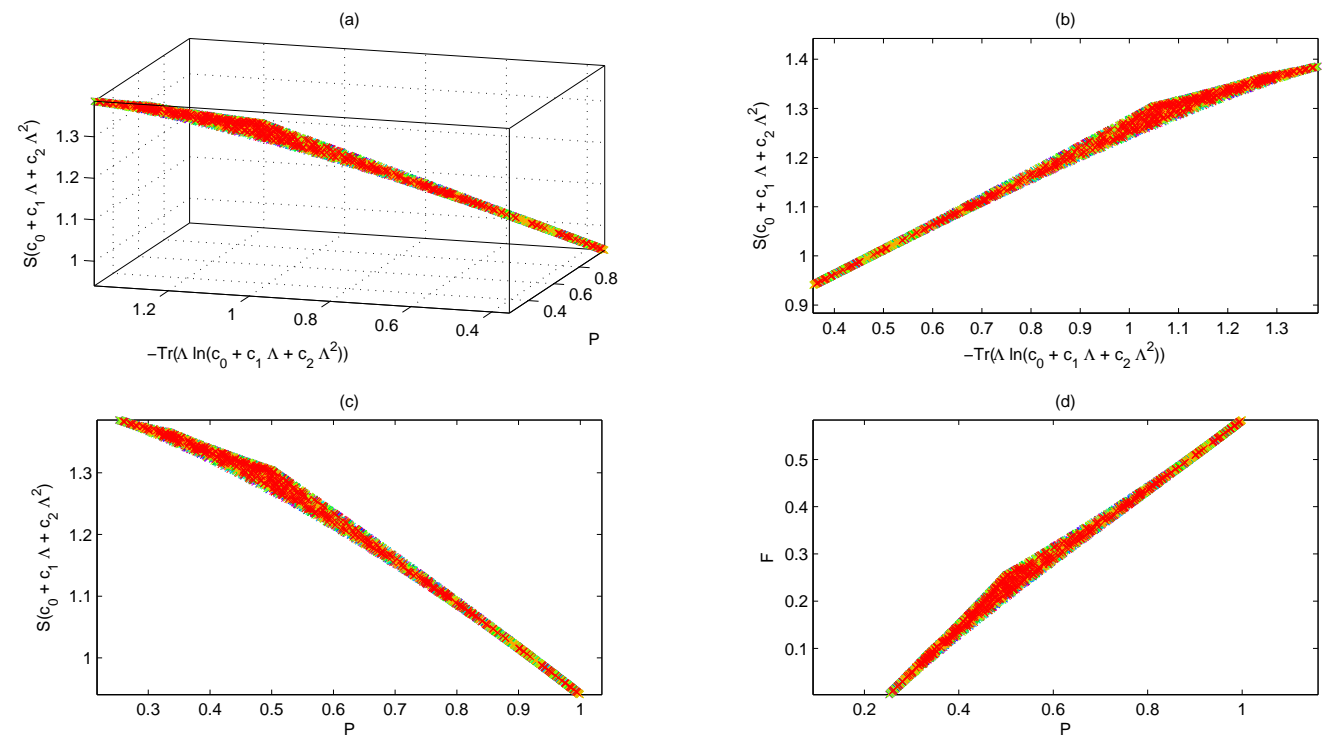

Figure 2: Two-qubit case $\left(d_{A}=d_{B}=2\right)$. (a) $3 \mathrm{D}$ scatter plot of $\left(\mathcal{P}(\Lambda),-\operatorname{Tr}\left(\Lambda \ln \left(c_{0}+c_{1} \Lambda+c_{2} \Lambda^{2}\right)\right), S\left(c_{0}+\right.\right.$ $\left.\left.c_{1} \Lambda+c_{2} \Lambda^{2}\right)\right)$. $2 \mathrm{D}$ scatter plots: (b) $-\operatorname{Tr}\left(\Lambda \ln \left(c_{0}+c_{1} \Lambda+c_{2} \Lambda^{2}\right)\right)$ vs. $\mathrm{S}\left(c_{0}+c_{1} \Lambda+c_{2} \Lambda^{2}\right)$; (c) $\mathcal{P}(\Lambda)$ vs. $\mathrm{S}\left(c_{0}+c_{1} \Lambda+c_{2} \Lambda^{2}\right) ;(\mathrm{d}) \mathcal{P}(\Lambda)$ vs. $\mathcal{F}(\Lambda)$.

Remark 3.5. Denote $\rho^{\prime}=U \rho U^{\dagger}$ for a fixed state $\rho$. If $U$ is such that $I(A: B)_{\rho^{\prime}}>I(A: B)_{\rho}$, then we say that $U$ enhances the correlation between $A$ and $B$, otherwise, we say that it weakens the correlation $A$ and $B$ when $I(A: B)_{\rho^{\prime}}<I(A: B)_{\rho}$. In particular, if $I(A: B)_{\rho^{\prime}}=0$, then we say that $U$ decouples $A$ 
from $B$. Clearly there exists a state (for example completely mixed state) of which its correlations cannot be enhanced by any unitary. We see from previous discussion, finding minimal mutual information is in terms of an optimization problem that is extremely hard to handle in higher dimensions. Proposition 3.2 and Theorem 3.4 can be seen as a different strategy based on probabilistic averages. Denote

$$
\Delta\left(\rho_{A B}\right):=\int I(A: B)_{\rho^{\prime}} \mathrm{d} \mu(U)-I(A: B)_{\rho} .
$$

If $\Delta(\rho)>0$, then the correlations existing in $\rho_{A B}$ can be enhanced by many unitaries (via a perspective of concentration of measure phenomenon from Measure Theory). Clearly a lot of states of product forms satisfies $\Delta(\rho)>0$. In the opposite, $\Delta(\rho)<0$ means that the correlations existing in $\rho_{A B}$ can be decreased by many unitaries. Naturally, some questions arise: For a given state $\rho_{A B}$, one wants to know wether if its unitary orbit of $\rho_{A B}$ contains product state(s).

Remark 3.6. A similar questions can be considered for the so-called quantum conditional mutual information (QCMI), defined by $I(A: B \mid E)_{\rho}:=\mathrm{S}\left(\rho_{A E}\right)+\mathrm{S}\left(\rho_{B E}\right)-\mathrm{S}\left(\rho_{A B E}\right)-\mathrm{S}\left(\rho_{E}\right)$ for a tripartite $\rho_{A B E}$ on a tensorial Hilbert space $\mathcal{H}_{A} \otimes \mathcal{H}_{B} \otimes \mathcal{H}_{E}$. We still denote $\rho_{A B E}^{\prime}=\left(U \otimes \mathbb{1}_{E}\right) \rho_{A B E}\left(U^{+} \otimes \mathbb{1}_{E}\right)$ for any unitary $U$ on $\mathcal{H}_{A} \otimes \mathcal{H}_{B}$. Analogously, maximal and/or minimal QCMI can be considered, that is,

$$
\max _{U} I(A: B \mid E)_{\rho^{\prime}} \quad \text { and } \quad \min _{U} I(A: B \mid E) \rho^{\prime} .
$$

Clearly $\min _{U} I(A: B \mid E)_{\rho^{\prime}}$ is very important since it gives a lower bound for QCMI:

$$
I(A: B \mid E)_{\rho} \geqslant \min _{U} I(A: B \mid E)_{\rho^{\prime}} .
$$

We can also consider the average QCMI:

$$
\int I(A: B \mid E)_{\rho^{\prime}} \mathrm{d} \mu(U) .
$$

All problems mentioned above are beyond the goal of this paper. We will come back to them in the future research.

Remark 3.7. Given a quantum channel $\mathcal{E}$ (trace-preserving and completely positive linear map) which is, via Kraus representation, represented as $\mathcal{E}=\sum_{j} \operatorname{Ad}_{E_{j}}$, where $\operatorname{Ad}_{E_{j}}(X):=E_{j} X E_{j}^{\dagger}$. We can use Lemma 2.1 to get the average purity of a unitary orbit of a given state undergoing a fixed quantum channel $\mathcal{E}$. Indeed, by Choi-Jamiołkowski isomorphism [2], $J(\mathcal{E}):=(\mathcal{E} \otimes \mathbb{1})\left(\left|\mathbb{1}_{d}\right\rangle\left\langle\mathbb{1}_{d}\right|\right)=\sum_{j}\left|E_{j}\right\rangle\left\langle E_{j}\right|$,

$$
\begin{aligned}
\int \mathcal{E}\left(U \rho U^{\dagger}\right)^{2} \mathrm{~d} \mu(U) & =\sum_{i, j} E_{i}\left(U \rho U^{\dagger} E_{i}^{\dagger} E_{j} U \rho U^{\dagger}\right) E_{j}^{\dagger} \\
& =\frac{d \operatorname{Tr}\left(\rho^{2}\right)-1}{d\left(d^{2}-1\right)} \sum_{i, j} \operatorname{Tr}\left(E_{i}^{\dagger} E_{j}\right) E_{i} E_{j}^{\dagger}+\frac{d-\operatorname{Tr}\left(\rho^{2}\right)}{d\left(d^{2}-1\right)} \mathcal{E}\left(\mathbb{1}_{d}\right)^{2},
\end{aligned}
$$

implying that

$$
\int \operatorname{Tr}\left(\mathcal{E}\left(U \rho U^{\dagger}\right)^{2}\right) \mathrm{d} \mu(U)=\frac{d \operatorname{Tr}\left(\rho^{2}\right)-1}{d\left(d^{2}-1\right)} \operatorname{Tr}\left(J(\mathcal{E})^{2}\right)+\frac{d-\operatorname{Tr}\left(\rho^{2}\right)}{d\left(d^{2}-1\right)} \operatorname{Tr}\left(\mathcal{E}\left(\mathbb{1}_{d}\right)^{2}\right),
$$

where the lhs of the above last formula is just the average purity of a unitary orbit of a given state undergoing a fixed quantum channel $\mathcal{E}$. 


\section{Concluding remarks}

In this paper, we investigate the average entropy of a subsystem along a global unitary orbit of a given mixed bipartite state in the finite-dimensional space. Although it is still unable to derive the closedform compact formula for the mixed state case, compared with Page's formula, nevertheless we get an analytical lower bound for this average entropy for the mixed state case. In deriving this analytical lower bound, we obtain some useful by-products of independent interest, for instance, for a bipartite quantum system, maximally mixed state can be represented by a uniform probability mixing of tensor products of two marginal states at each point within the global unitary orbit of any given mixed bipartite state. This is amazing. In addition, from the discussion after finishing the proof of Proposition 3.2, we see that the average entropy of a subsystem is intimately related to the well-known "quantum marginal problem" or $N$-representability in quantum chemistry. Besides, it also connects with entanglement polytope, a notion proposed by M. Walter et al. in studying multipartite entanglement from single-particle information [21].

These obtained results can be applied to estimate average correlation along a global unitary orbit of a given mixed bipartite state. The corresponding numerics about these results is also provided in lower dimensional case (for instance $d_{A}=d_{B}=2$ ). Except that, the results obtained in the present paper can also be used to study further the average coherence of a class of random states induced from isospectral bipartite mixed states. Indeed, recently we have already calculated exactly the average coherence for random mixed quantum states $[23,26]$ induced from random bipartite pure states.

Finally, we conclude this section with two open problems: (i) computing the average coherence (via relative entropy of coherence) of a subsystem of isospectral bipartite systems

$$
\int\left(\mathrm{S}\left(\left(\rho_{A}^{\prime}\right)_{\operatorname{diag}}\right)-\mathrm{S}\left(\rho_{A}^{\prime}\right)\right) \mathrm{d} \mu(U),
$$

and (ii) proving the following identity for the balanced system, i.e., $d_{A}=d_{B}$,

$$
\int \mathrm{S}\left(\rho_{A}^{\prime}\right) \mathrm{d} \mu(U)=\int \mathrm{S}\left(\rho_{B}^{\prime}\right) \mathrm{d} \mu(U) .
$$

If (4.2) were true, then we would see from Theorem 2.2 and Theorem 3.4 that

$$
-\ln \left(1-a_{1}\right) \leqslant \int \mathrm{S}\left(\rho_{A}^{\prime}\right) \mathrm{d} \mu(U) \leqslant \frac{1}{2} \mathrm{~S}\left(c_{0} \cdot \mathbb{1}_{d}+c_{1} \cdot \rho_{A B}+c_{2} \cdot \rho_{A B}^{2}\right) .
$$

We hope that the present work and questions proposed can bring out more interesting and insightful perspective(s) in quantum information theory.

\section{Acknowledgements}

L. Zhang is supported Natural Science Foundation of Zhejiang Province of China (LY17A010027) and also by National Natural Science Foundation of China (Nos.11301124 \& 61673145). H. Xiang is supported by the National Natural Science Foundation of China (Nos.11571265 \& 11471253). Michael Walter is also acknowledged for his comments on this manuscript. 


\section{Appendix}

\section{A. The proof of Lemma 2.1}

In this paper, we will utilize some notion of matrix integral $[4,5,22]$. The formula in Lemma 2.1 is given firstly. A detailed reasoning is presented here.

The proof of Lemma 2.1. Firstly we note that

$$
\begin{aligned}
\left\langle i_{1}\left|U A U^{\dagger} B U X U^{\dagger} C U D U^{\dagger}\right| i_{1}^{\prime}\right\rangle=\sum_{j_{1}, j_{1}^{\prime}}\left\langle i_{1}|U| j_{1}\right\rangle\left\langle j_{1}\left|A U^{\dagger} B U X U^{\dagger} C U D\right| j_{1}^{\prime}\right\rangle\left\langle j_{1}^{\prime}\left|U^{\dagger}\right| i_{1}^{\prime}\right\rangle \\
=\sum_{i_{2}, j_{1}, j_{2}, i_{2}^{\prime}, j_{1}^{\prime}, j_{2}^{\prime}} U_{i_{1} j_{1}} \bar{U}_{i_{1}^{\prime} j_{1}^{\prime}}\left\langle j_{1}|A| j_{2}^{\prime}\right\rangle\left\langle j_{2}^{\prime}\left|U^{\dagger}\right| i_{2}^{\prime}\right\rangle\left\langle i_{2}^{\prime}\left|B U X U^{\dagger} C\right| i_{2}\right\rangle\left\langle i_{2}|U| j_{2}\right\rangle\left\langle j_{2}|D| j_{1}^{\prime}\right\rangle \\
=\sum_{i_{2}, j_{1}, j_{2}, i_{2}^{\prime}, j_{1}^{\prime}, j_{2}^{\prime}} U_{i_{1} j_{1}} U_{i_{2} j_{2}} \bar{U}_{i_{1}^{\prime} j_{1}^{\prime}} \bar{U}_{i_{2}^{\prime} j_{2}^{\prime}}\left\langle j_{1}|A| j_{2}^{\prime}\right\rangle\left\langle i_{2}^{\prime}\left|B U X U^{\dagger} C\right| i_{2}\right\rangle\left\langle j_{2}|D| j_{1}^{\prime}\right\rangle \\
=\sum_{i_{2}, i_{3}, j_{1}, j_{2}, j_{3}, i_{2}^{\prime}, i_{3}^{\prime}, j_{1}^{\prime}, j_{2}^{\prime}, j_{3}^{\prime}} U_{i_{1} j_{1}} U_{i_{2} j_{2}} \bar{U}_{i_{1}^{\prime} j_{1}^{\prime}} \bar{U}_{i_{2}^{\prime} j_{2}^{\prime}} \\
\quad \times\left\langle j_{1}|A| j_{2}^{\prime}\right\rangle\left\langle i_{2}^{\prime}|B| i_{3}\right\rangle\left\langle i_{3}|U| j_{3}\right\rangle\left\langle j_{3}|X| j_{3}^{\prime}\right\rangle\left\langle j_{3}^{\prime}\left|U^{\dagger}\right| i_{3}^{\prime}\right\rangle\left\langle i_{3}^{\prime}|C| i_{2}\right\rangle\left\langle j_{2}|D| j_{1}^{\prime}\right\rangle \\
=\sum_{i_{2}, i_{3}, j_{1}, j_{2}, j_{3}, i_{2}^{\prime}, i_{3}, j_{1}^{\prime}, j_{2}^{\prime}, j_{3}^{\prime}} U_{i_{1} j_{1}} U_{i_{2} j_{2}} U_{i_{3} j_{3}} \bar{U}_{i_{1}^{\prime} j_{1}^{\prime}} \bar{U}_{i_{2}^{\prime} j_{2}^{\prime}} \bar{U}_{i_{3}^{\prime} j_{3}^{\prime}}\left\langle j_{1}|A| j_{2}^{\prime}\right\rangle\left\langle i_{2}^{\prime}|B| i_{3}\right\rangle\left\langle j_{3}|X| j_{3}^{\prime}\right\rangle\left\langle i_{3}^{\prime}|C| i_{2}\right\rangle\left\langle j_{2}|D| j_{1}^{\prime}\right\rangle .
\end{aligned}
$$

Then we have:

$$
\begin{aligned}
& \left\langle i_{1}\left|\int U A U^{\dagger} B U X U^{\dagger} C U D U^{\dagger} d U\right| i_{1}^{\prime}\right\rangle \\
& =\sum_{i_{2}, i_{3}, j_{1}, j_{2}, j_{3}, i_{2}^{\prime}, i_{3}^{\prime}, j_{1}^{\prime}, j_{2}^{\prime}, j_{3}^{\prime}} A_{j_{1}, j_{2}^{\prime}} B_{i_{2}^{\prime}, i_{3}} X_{j_{3}, j_{3}^{\prime}} C_{i_{3}^{\prime}, i_{2}} D_{j_{2}, j_{1}^{\prime}}\left(\int U_{i_{1} j_{1}} U_{i_{2} j_{2}} U_{i_{3} j_{3}} \bar{U}_{i_{1}^{\prime} j_{1}^{\prime}} \bar{U}_{i_{2}^{\prime} j_{2}^{\prime}} \bar{U}_{i_{3}^{\prime} j_{3}^{\prime}} d U\right) \\
& =\sum_{i_{2}, i_{3}, j_{1}, j_{2}, j_{3}, i_{2}^{\prime}, i_{3}^{\prime}, j_{1}^{\prime}, j_{2}^{\prime}, j_{3}^{\prime}} A_{j_{1}, j_{2}^{\prime}} B_{i_{2}^{\prime}, i_{3}} X_{j_{3}, j_{3}^{\prime}} C_{i_{3}^{\prime}, i_{2}} D_{j_{2}, j_{1}^{\prime}} \\
& \times\left(\sum_{\pi, \sigma \in S_{3}}\left\langle i_{1} \mid i_{\pi(1)}^{\prime}\right\rangle\left\langle i_{2} \mid i_{\pi(2)}^{\prime}\right\rangle\left\langle i_{3} \mid i_{\pi(3)}^{\prime}\right\rangle\left\langle j_{1} \mid j_{\sigma(1)}^{\prime}\right\rangle\left\langle j_{2} \mid j_{\sigma(2)}^{\prime}\right\rangle\left\langle j_{3} \mid j_{\sigma(3)}^{\prime}\right\rangle \mathrm{Wg}\left(\sigma \pi^{-1}\right)\right) \\
& =\sum_{\pi, \sigma \in S_{3}} \operatorname{Wg}\left(\sigma \pi^{-1}\right) \\
& \times\left(\sum_{i_{2}, i_{3}, j_{1}, j_{2}, j_{3}, i_{2}^{\prime}, i_{3}^{\prime}, j_{1}^{\prime}, j_{2}^{\prime}, j_{3}^{\prime}} A_{j_{1}, j_{2}^{\prime}} B_{i_{2}, i_{3}} X_{j_{3}, j_{3}^{\prime}} C_{i_{3}^{\prime}, i_{2}} D_{j_{2}, j_{1}^{\prime}}\left\langle i_{1} \mid i_{\pi(1)}^{\prime}\right\rangle\left\langle i_{2} \mid i_{\pi(2)}^{\prime}\right\rangle\left\langle i_{3} \mid i_{\pi(3)}^{\prime}\right\rangle\left\langle j_{1} \mid j_{\sigma(1)}^{\prime}\right\rangle\left\langle j_{2} \mid j_{\sigma(2)}^{\prime}\right\rangle\left\langle j_{3} \mid j_{\sigma(3)}^{\prime}\right\rangle\right) .
\end{aligned}
$$

In what follows, we compute this value step-by-step.

(1). If $(\pi, \sigma)=((1),(1))$, then

$$
\begin{aligned}
& \sum_{i_{2}, i_{3}, j_{1}, j_{2}, j_{3}, i_{2}^{\prime}, i_{3}, j_{1}^{\prime}, j_{2}^{\prime}, j_{3}^{\prime}} A_{j_{1}, j_{2}^{\prime}} B_{i_{2}^{\prime}, i_{3}} X_{j_{3}, j_{3}^{\prime}} C_{i_{3}^{\prime}, i_{2}} D_{j_{2}, j_{1}^{\prime}}\left\langle i_{1} \mid i_{1}^{\prime}\right\rangle\left\langle i_{2} \mid i_{2}^{\prime}\right\rangle\left\langle i_{3} \mid i_{3}^{\prime}\right\rangle\left\langle j_{1} \mid j_{1}^{\prime}\right\rangle\left\langle j_{2} \mid j_{2}^{\prime}\right\rangle\left\langle j_{3} \mid j_{3}^{\prime}\right\rangle \\
& =\operatorname{Tr}(A D) \operatorname{Tr}(X) \operatorname{Tr}(B C)\left\langle i_{1} \mid i_{1}^{\prime}\right\rangle .
\end{aligned}
$$


(2). If $(\pi, \sigma)=((1),(12))$, then

$$
\begin{aligned}
& \sum_{i_{2}, i_{3}, j_{1}, j_{2}, j_{3}, i_{2}^{\prime}, i_{3}^{\prime}, j_{1}^{\prime}, j_{2}^{\prime}, j_{3}^{\prime}} A_{j_{1}, j_{2}^{\prime}} B_{i_{2}^{\prime}, i_{3}} X_{j_{3}, j_{3}^{\prime}} C_{i_{3}^{\prime}, i_{2}} D_{j_{2}, j_{1}^{\prime}}\left\langle i_{1} \mid i_{1}^{\prime}\right\rangle\left\langle i_{2} \mid i_{2}^{\prime}\right\rangle\left\langle i_{3} \mid i_{3}^{\prime}\right\rangle\left\langle j_{1} \mid j_{2}^{\prime}\right\rangle\left\langle j_{2} \mid j_{1}^{\prime}\right\rangle\left\langle j_{3} \mid j_{3}^{\prime}\right\rangle \\
& =\operatorname{Tr}(A) \operatorname{Tr}(D) \operatorname{Tr}(X) \operatorname{Tr}(B C)\left\langle i_{1} \mid i_{1}^{\prime}\right\rangle .
\end{aligned}
$$

(3). If $(\pi, \sigma)=((1),(13))$, then

$$
\begin{aligned}
& \sum_{i_{2}, i_{3}, j_{1}, j_{2}, j_{3}, i_{2}^{\prime}, i_{3}^{\prime}, j_{1}^{\prime}, j_{2}^{\prime}, j_{3}^{\prime}} A_{j_{1}, j_{2}^{\prime}} B_{i_{2}^{\prime}, i_{3}} X_{j_{3}, j_{3}^{\prime}} C_{i_{3}^{\prime}, i_{2}} D_{j_{2}, j_{1}^{\prime}}\left\langle i_{1} \mid i_{1}^{\prime}\right\rangle\left\langle i_{2} \mid i_{2}^{\prime}\right\rangle\left\langle i_{3} \mid i_{3}^{\prime}\right\rangle\left\langle j_{1} \mid j_{3}^{\prime}\right\rangle\left\langle j_{2} \mid j_{2}^{\prime}\right\rangle\left\langle j_{3} \mid j_{1}^{\prime}\right\rangle \\
& =\operatorname{Tr}(A D X) \operatorname{Tr}(B C)\left\langle i_{1} \mid i_{1}^{\prime}\right\rangle .
\end{aligned}
$$

(4). If $(\pi, \sigma)=((1),(23))$, then

$$
\begin{aligned}
& \sum_{i_{2}, i_{3}, j_{1}, j_{2}, j_{3}, i_{2}^{\prime}, i_{3}^{\prime}, j_{1}^{\prime}, j_{2}^{\prime}, j_{3}^{\prime}} A_{j_{1}, j_{2}^{\prime}} B_{i_{2}^{\prime}, i_{3}} X_{j_{3}, j_{3}^{\prime}} C_{i_{3}^{\prime}, i_{2}} D_{j_{2}, j_{1}^{\prime}}\left\langle i_{1} \mid i_{1}^{\prime}\right\rangle\left\langle i_{2} \mid i_{2}^{\prime}\right\rangle\left\langle i_{3} \mid i_{3}^{\prime}\right\rangle\left\langle j_{1} \mid j_{1}^{\prime}\right\rangle\left\langle j_{2} \mid j_{3}^{\prime}\right\rangle\left\langle j_{3} \mid j_{2}^{\prime}\right\rangle \\
& =\operatorname{Tr}(D A X) \operatorname{Tr}(B C)\left\langle i_{1} \mid i_{1}^{\prime}\right\rangle .
\end{aligned}
$$

(5). If $(\pi, \sigma)=((1),(123))$, then

$$
\begin{aligned}
& \sum_{i_{2}, i_{3}, j_{1}, j_{2}, j_{3}, i_{2}^{\prime}, i_{3}^{\prime}, j_{1}^{\prime}, j_{2}^{\prime}, j_{3}^{\prime}} A_{j_{1}, j_{2}^{\prime}} B_{i_{2}^{\prime}, i_{3}} X_{j_{3}, j_{3}^{\prime}} C_{i_{3}^{\prime}, i_{2}} D_{j_{2}, j_{1}^{\prime}}\left\langle i_{1} \mid i_{1}^{\prime}\right\rangle\left\langle i_{2} \mid i_{2}^{\prime}\right\rangle\left\langle i_{3} \mid i_{3}^{\prime}\right\rangle\left\langle j_{1} \mid j_{2}^{\prime}\right\rangle\left\langle j_{2} \mid j_{3}^{\prime}\right\rangle\left\langle j_{3} \mid j_{1}^{\prime}\right\rangle \\
& =\operatorname{Tr}(A) \operatorname{Tr}(D X) \operatorname{Tr}(B C)\left\langle i_{1} \mid i_{1}^{\prime}\right\rangle .
\end{aligned}
$$

(6). If $(\pi, \sigma)=((1),(132))$, then

$$
\begin{aligned}
& \sum_{i_{2}, i_{3}, j_{1}, j_{2}, j_{3}, i_{2}^{\prime}, i_{3}^{\prime}, j_{1}^{\prime}, j_{2}^{\prime}, j_{3}^{\prime}} A_{j_{1}, j_{2}^{\prime}} B_{i_{2}^{\prime}, i_{3}} X_{j_{3}, j_{3}^{\prime}} C_{i_{3}^{\prime}, i_{2}} D_{j_{2}, j_{1}^{\prime}}\left\langle i_{1} \mid i_{1}^{\prime}\right\rangle\left\langle i_{2} \mid i_{2}^{\prime}\right\rangle\left\langle i_{3} \mid i_{3}^{\prime}\right\rangle\left\langle j_{1} \mid j_{3}^{\prime}\right\rangle\left\langle j_{2} \mid j_{1}^{\prime}\right\rangle\left\langle j_{3} \mid j_{2}^{\prime}\right\rangle \\
& =\operatorname{Tr}(D) \operatorname{Tr}(A X) \operatorname{Tr}(B C)\left\langle i_{1} \mid i_{1}^{\prime}\right\rangle .
\end{aligned}
$$

(7). If $(\pi, \sigma)=((12),(1))$, then

$$
\begin{aligned}
& \sum_{i_{2}, i_{3}, j_{1}, j_{2}, j_{3}, i_{2}^{\prime}, i_{3}, j_{1}^{\prime}, j_{2}^{\prime}, j_{3}^{\prime}} A_{j_{1}, j_{2}^{\prime}} B_{i_{2}^{\prime}, i_{3}} X_{j_{3}, j_{3}^{\prime}} C_{i_{3}^{\prime}, i_{2}} D_{j_{2}, j_{1}^{\prime}}\left\langle i_{1} \mid i_{2}^{\prime}\right\rangle\left\langle i_{2} \mid i_{1}^{\prime}\right\rangle\left\langle i_{3} \mid i_{3}^{\prime}\right\rangle\left\langle j_{1} \mid j_{1}^{\prime}\right\rangle\left\langle j_{2} \mid j_{2}^{\prime}\right\rangle\left\langle j_{3} \mid j_{3}^{\prime}\right\rangle \\
& =\operatorname{Tr}(A D) \operatorname{Tr}(X)\left\langle i_{1}|B C| i_{1}^{\prime}\right\rangle .
\end{aligned}
$$

(8). If $(\pi, \sigma)=((12),(12))$, then

$$
\begin{aligned}
& \sum_{i_{2}, i_{3}, j_{1}, j_{2}, j_{3}, i_{2}^{\prime}, i_{3}^{\prime}, j_{1}^{\prime}, j_{2}^{\prime}, j_{3}^{\prime}} A_{j_{1}, j_{2}^{\prime}} B_{i_{2}^{\prime}, i_{3}} X_{j_{3}, j_{3}^{\prime}} C_{i_{3}^{\prime}, i_{2}} D_{j_{2}, j_{1}^{\prime}}\left\langle i_{1} \mid i_{2}^{\prime}\right\rangle\left\langle i_{2} \mid i_{1}^{\prime}\right\rangle\left\langle i_{3} \mid i_{3}^{\prime}\right\rangle\left\langle j_{1} \mid j_{2}^{\prime}\right\rangle\left\langle j_{2} \mid j_{1}^{\prime}\right\rangle\left\langle j_{3} \mid j_{3}^{\prime}\right\rangle \\
& =\operatorname{Tr}(A) \operatorname{Tr}(D) \operatorname{Tr}(X)\left\langle i_{1}|B C| i_{1}^{\prime}\right\rangle .
\end{aligned}
$$

(9). If $(\pi, \sigma)=((12),(13))$, then

$$
\begin{aligned}
& \sum_{i_{2}, i_{3}, j_{1}, j_{2}, j_{3}, i_{2}^{\prime}, i_{3}^{\prime}, j_{1}^{\prime}, j_{2}^{\prime}, j_{3}^{\prime}} A_{j_{1}, j_{2}^{\prime}} B_{i_{2}^{\prime}, i_{3}} X_{j_{3}, j_{3}^{\prime}} C_{i_{3}^{\prime}, i_{2}} D_{j_{2}, j_{1}^{\prime}}\left\langle i_{1} \mid i_{2}^{\prime}\right\rangle\left\langle i_{2} \mid i_{1}^{\prime}\right\rangle\left\langle i_{3} \mid i_{3}^{\prime}\right\rangle\left\langle j_{1} \mid j_{3}^{\prime}\right\rangle\left\langle j_{2} \mid j_{2}^{\prime}\right\rangle\left\langle j_{3} \mid j_{1}^{\prime}\right\rangle \\
& =\operatorname{Tr}(A D X)\left\langle i_{1}|B C| i_{1}^{\prime}\right\rangle .
\end{aligned}
$$

(10). If $(\pi, \sigma)=((12),(23))$, then

$$
\begin{aligned}
& \sum_{i_{2}, i_{3}, j_{1}, j_{2}, j_{3}, i_{2}^{\prime}, i_{3}^{\prime}, j_{1}^{\prime}, j_{2}^{\prime}, j_{3}^{\prime}} A_{j_{1}, j_{2}^{\prime}} B_{i_{2}^{\prime}, i_{3}} X_{j_{3}, j_{3}^{\prime}} C_{i_{3}^{\prime}, i_{2}} D_{j_{2}, j_{1}^{\prime}}\left\langle i_{1} \mid i_{2}^{\prime}\right\rangle\left\langle i_{2} \mid i_{1}^{\prime}\right\rangle\left\langle i_{3} \mid i_{3}^{\prime}\right\rangle\left\langle j_{1} \mid j_{1}^{\prime}\right\rangle\left\langle j_{2} \mid j_{3}^{\prime}\right\rangle\left\langle j_{3} \mid j_{2}^{\prime}\right\rangle \\
& =\operatorname{Tr}(D A X)\left\langle i_{1}|B C| i_{1}^{\prime}\right\rangle .
\end{aligned}
$$


(11). If $(\pi, \sigma)=((12),(123))$, then

$$
\begin{aligned}
& \sum_{i_{2}, i_{3}, j_{1}, j_{2}, j_{3}, i_{2}^{\prime}, i_{3}^{\prime}, j_{1}^{\prime}, j_{2}^{\prime}, j_{3}^{\prime}} A_{j_{1}, j_{2}^{\prime}} B_{i_{2}^{\prime}, i_{3}} X_{j_{3}, j_{3}^{\prime}} C_{i_{3}^{\prime}, i_{2}} D_{j_{2}, j_{1}^{\prime}}\left\langle i_{1} \mid i_{2}^{\prime}\right\rangle\left\langle i_{2} \mid i_{1}^{\prime}\right\rangle\left\langle i_{3} \mid i_{3}^{\prime}\right\rangle\left\langle j_{1} \mid j_{2}^{\prime}\right\rangle\left\langle j_{2} \mid j_{3}^{\prime}\right\rangle\left\langle j_{3} \mid j_{1}^{\prime}\right\rangle \\
& =\operatorname{Tr}(A) \operatorname{Tr}(D X)\left\langle i_{1}|B C| i_{1}^{\prime}\right\rangle .
\end{aligned}
$$

(12). If $(\pi, \sigma)=((12),(132))$, then

$$
\begin{aligned}
& \sum_{i_{2}, i_{3}, j_{1}, j_{2}, j_{3}, i_{2}^{\prime}, i_{3}^{\prime}, j_{1}^{\prime}, j_{2}^{\prime}, j_{3}^{\prime}} A_{j_{1}, j_{2}^{\prime}} B_{i_{2}^{\prime}, i_{3}} X_{j_{3}, j_{3}^{\prime}} C_{i_{3}^{\prime}, i_{2}} D_{j_{2}, j_{1}^{\prime}}\left\langle i_{1} \mid i_{2}^{\prime}\right\rangle\left\langle i_{2} \mid i_{1}^{\prime}\right\rangle\left\langle i_{3} \mid i_{3}^{\prime}\right\rangle\left\langle j_{1} \mid j_{3}^{\prime}\right\rangle\left\langle j_{2} \mid j_{1}^{\prime}\right\rangle\left\langle j_{3} \mid j_{2}^{\prime}\right\rangle \\
& =\operatorname{Tr}(D) \operatorname{Tr}(A X)\left\langle i_{1}|B C| i_{1}^{\prime}\right\rangle .
\end{aligned}
$$

(13). If $(\pi, \sigma)=((13),(1))$, then

$$
\begin{aligned}
& \sum_{i_{2}, i_{3}, j_{1}, j_{2}, j_{3}, i_{2}^{\prime}, i_{3}^{\prime}, j_{1}^{\prime}, j_{2}^{\prime}, j_{3}^{\prime}} A_{j_{1}, j_{2}^{\prime}} B_{i_{2}^{\prime}, i_{3}} X_{j_{3}, j_{3}^{\prime}} C_{i_{3}^{\prime}, i_{2}} D_{j_{2}, j_{1}^{\prime}}\left\langle i_{1} \mid i_{3}^{\prime}\right\rangle\left\langle i_{2} \mid i_{2}^{\prime}\right\rangle\left\langle i_{3} \mid i_{1}^{\prime}\right\rangle\left\langle j_{1} \mid j_{1}^{\prime}\right\rangle\left\langle j_{2} \mid j_{2}^{\prime}\right\rangle\left\langle j_{3} \mid j_{3}^{\prime}\right\rangle \\
& =\operatorname{Tr}(A D) \operatorname{Tr}(X)\left\langle i_{1}|C B| i_{1}^{\prime}\right\rangle .
\end{aligned}
$$

(14). If $(\pi, \sigma)=((13),(12))$, then

$$
\begin{aligned}
& \sum_{i_{2}, i_{3}, j_{1}, j_{2}, j_{3}, i_{2}^{\prime}, i_{3}^{\prime}, j_{1}^{\prime}, j_{2}^{\prime}, j_{3}^{\prime}} A_{j_{1}, j_{2}^{\prime}} B_{i_{2}^{\prime}, i_{3}} X_{j_{3}, j_{3}^{\prime}} C_{i_{3}^{\prime}, i_{2}} D_{j_{2}, j_{1}^{\prime}}\left\langle i_{1} \mid i_{3}^{\prime}\right\rangle\left\langle i_{2} \mid i_{2}^{\prime}\right\rangle\left\langle i_{3} \mid i_{1}^{\prime}\right\rangle\left\langle j_{1} \mid j_{2}^{\prime}\right\rangle\left\langle j_{2} \mid j_{1}^{\prime}\right\rangle\left\langle j_{3} \mid j_{3}^{\prime}\right\rangle \\
& =\operatorname{Tr}(A) \operatorname{Tr}(D) \operatorname{Tr}(X)\left\langle i_{1}|C B| i_{1}^{\prime}\right\rangle .
\end{aligned}
$$

(15). If $(\pi, \sigma)=((13),(13))$, then

$$
\begin{aligned}
& \sum_{i_{2}, i_{3}, j_{1}, j_{2}, j_{3}, i_{2}^{\prime}, i_{3}^{\prime}, j_{1}^{\prime}, j_{2}^{\prime}, j_{3}^{\prime}} A_{j_{1}, j_{2}^{\prime}} B_{i_{2}^{\prime}, i_{3}} X_{j_{3}, j_{3}^{\prime}} C_{i_{3}^{\prime}, i_{2}} D_{j_{2}, j_{1}^{\prime}}\left\langle i_{1} \mid i_{3}^{\prime}\right\rangle\left\langle i_{2} \mid i_{2}^{\prime}\right\rangle\left\langle i_{3} \mid i_{1}^{\prime}\right\rangle\left\langle j_{1} \mid j_{3}^{\prime}\right\rangle\left\langle j_{2} \mid j_{2}^{\prime}\right\rangle\left\langle j_{3} \mid j_{1}^{\prime}\right\rangle \\
& =\operatorname{Tr}(A D X)\left\langle i_{1}|C B| i_{1}^{\prime}\right\rangle .
\end{aligned}
$$

(16). If $(\pi, \sigma)=((13),(23))$, then

$$
\begin{aligned}
& \sum_{i_{2}, i_{3}, j_{1}, j_{2}, j_{3}, i_{2}^{\prime}, i_{3}^{\prime}, j_{1}^{\prime}, j_{2}^{\prime}, j_{3}^{\prime}} A_{j_{1}, j_{2}^{\prime}} B_{i_{2}^{\prime}, i_{3}} X_{j_{3}, j_{3}^{\prime}} C_{i_{3}^{\prime}, i_{2}} D_{j_{2}, j_{1}^{\prime}}\left\langle i_{1} \mid i_{3}^{\prime}\right\rangle\left\langle i_{2} \mid i_{2}^{\prime}\right\rangle\left\langle i_{3} \mid i_{1}^{\prime}\right\rangle\left\langle j_{1} \mid j_{1}^{\prime}\right\rangle\left\langle j_{2} \mid j_{3}^{\prime}\right\rangle\left\langle j_{3} \mid j_{2}^{\prime}\right\rangle \\
& =\operatorname{Tr}(D A X)\left\langle i_{1}|C B| i_{1}^{\prime}\right\rangle .
\end{aligned}
$$

(17). If $(\pi, \sigma)=((13),(123))$, then

$$
\begin{aligned}
& \sum_{i_{2}, i_{3}, j_{1}, j_{2}, j_{3}, i_{2}^{\prime}, i_{3}^{\prime}, j_{1}^{\prime}, j_{2}^{\prime}, j_{3}^{\prime}} A_{j_{1}, j_{2}^{\prime}} B_{i_{2}^{\prime}, i_{3}} X_{j_{3}, j_{3}^{\prime}} C_{i_{3}^{\prime}, i_{2}} D_{j_{2}, j_{1}^{\prime}}\left\langle i_{1} \mid i_{3}^{\prime}\right\rangle\left\langle i_{2} \mid i_{2}^{\prime}\right\rangle\left\langle i_{3} \mid i_{1}^{\prime}\right\rangle\left\langle j_{1} \mid j_{2}^{\prime}\right\rangle\left\langle j_{2} \mid j_{3}^{\prime}\right\rangle\left\langle j_{3} \mid j_{1}^{\prime}\right\rangle \\
& =\operatorname{Tr}(A) \operatorname{Tr}(D X)\left\langle i_{1}|C B| i_{1}^{\prime}\right\rangle .
\end{aligned}
$$

(18). If $(\pi, \sigma)=((13),(132))$, then

$$
\begin{aligned}
& \sum_{i_{2}, i_{3}, j_{1}, j_{2}, j_{3}, i_{2}^{\prime}, i_{3}^{\prime}, j_{1}^{\prime}, j_{2}^{\prime}, j_{3}^{\prime}} A_{j_{1}, j_{2}^{\prime}} B_{i_{2}^{\prime}, i_{3}} X_{j_{3}, j_{3}^{\prime}} C_{i_{3}^{\prime}, i_{2}} D_{j_{2}, j_{1}^{\prime}}\left\langle i_{1} \mid i_{3}^{\prime}\right\rangle\left\langle i_{2} \mid i_{2}^{\prime}\right\rangle\left\langle i_{3} \mid i_{1}^{\prime}\right\rangle\left\langle j_{1} \mid j_{3}^{\prime}\right\rangle\left\langle j_{2} \mid j_{1}^{\prime}\right\rangle\left\langle j_{3} \mid j_{2}^{\prime}\right\rangle \\
& =\operatorname{Tr}(D) \operatorname{Tr}(A X)\left\langle i_{1}|C B| i_{1}^{\prime}\right\rangle .
\end{aligned}
$$

(19). If $(\pi, \sigma)=((23),(1))$, then

$$
\begin{aligned}
& \sum_{i_{2}, i_{3}, j_{1}, j_{2}, j_{3}, i_{2}^{\prime}, i_{3}^{\prime}, j_{1}^{\prime}, j_{2}^{\prime}, j_{3}^{\prime}} A_{j_{1}, j_{2}^{\prime}} B_{i_{2}^{\prime}, i_{3}} X_{j_{3}, j_{3}^{\prime}} C_{i_{3}^{\prime}, i_{2}} D_{j_{2}, j_{1}^{\prime}}\left\langle i_{1} \mid i_{1}^{\prime}\right\rangle\left\langle i_{2} \mid i_{3}^{\prime}\right\rangle\left\langle i_{3} \mid i_{2}^{\prime}\right\rangle\left\langle j_{1} \mid j_{1}^{\prime}\right\rangle\left\langle j_{2} \mid j_{2}^{\prime}\right\rangle\left\langle j_{3} \mid j_{3}^{\prime}\right\rangle \\
& =\operatorname{Tr}(A D) \operatorname{Tr}(X) \operatorname{Tr}(B) \operatorname{Tr}(C)\left\langle i_{1} \mid i_{1}^{\prime}\right\rangle .
\end{aligned}
$$


(20). If $(\pi, \sigma)=((23),(12))$, then

$$
\begin{aligned}
& \sum_{i_{2}, i_{3}, j_{1}, j_{2}, j_{3}, i_{2}^{\prime}, i_{3}^{\prime}, j_{1}^{\prime}, j_{2}^{\prime}, j_{3}^{\prime}} A_{j_{1}, j_{2}^{\prime}} B_{i_{2}^{\prime}, i_{3}} X_{j_{3}, j_{3}^{\prime}} C_{i_{3}^{\prime}, i_{2}} D_{j_{2}, j_{1}^{\prime}}\left\langle i_{1} \mid i_{1}^{\prime}\right\rangle\left\langle i_{2} \mid i_{3}^{\prime}\right\rangle\left\langle i_{3} \mid i_{2}^{\prime}\right\rangle\left\langle j_{1} \mid j_{2}^{\prime}\right\rangle\left\langle j_{2} \mid j_{1}^{\prime}\right\rangle\left\langle j_{3} \mid j_{3}^{\prime}\right\rangle \\
& =\operatorname{Tr}(A) \operatorname{Tr}(D) \operatorname{Tr}(X) \operatorname{Tr}(B) \operatorname{Tr}(C)\left\langle i_{1} \mid i_{1}^{\prime}\right\rangle .
\end{aligned}
$$

(21). If $(\pi, \sigma)=((23),(13))$, then

$$
\begin{aligned}
& \sum_{i_{2}, i_{3}, j_{1}, j_{2}, j_{3}, i_{2}^{\prime}, i_{3}^{\prime}, j_{1}^{\prime}, j_{2}^{\prime}, j_{3}^{\prime}} A_{j_{1}, j_{2}^{\prime}} B_{i_{2}^{\prime}, i_{3}} X_{j_{3}, j_{3}^{\prime}} C_{i_{3}^{\prime}, i_{2}} D_{j_{2}, j_{1}^{\prime}}\left\langle i_{1} \mid i_{1}^{\prime}\right\rangle\left\langle i_{2} \mid i_{3}^{\prime}\right\rangle\left\langle i_{3} \mid i_{2}^{\prime}\right\rangle\left\langle j_{1} \mid j_{3}^{\prime}\right\rangle\left\langle j_{2} \mid j_{2}^{\prime}\right\rangle\left\langle j_{3} \mid j_{1}^{\prime}\right\rangle \\
& =\operatorname{Tr}(A D X) \operatorname{Tr}(B) \operatorname{Tr}(C)\left\langle i_{1} \mid i_{1}^{\prime}\right\rangle .
\end{aligned}
$$

(22). If $(\pi, \sigma)=((23),(23))$, then

$$
\begin{aligned}
& \sum_{i_{2}, i_{3}, j_{1}, j_{2}, j_{3}, i_{2}^{\prime}, i_{3}^{\prime}, j_{1}^{\prime}, j_{2}^{\prime}, j_{3}^{\prime}} A_{j_{1}, j_{2}^{\prime}} B_{i_{2}^{\prime}, i_{3}} X_{j_{3}, j_{3}^{\prime}} C_{i_{3}^{\prime}, i_{2}} D_{j_{2}, j_{1}^{\prime}}\left\langle i_{1} \mid i_{1}^{\prime}\right\rangle\left\langle i_{2} \mid i_{3}^{\prime}\right\rangle\left\langle i_{3} \mid i_{2}^{\prime}\right\rangle\left\langle j_{1} \mid j_{1}^{\prime}\right\rangle\left\langle j_{2} \mid j_{3}^{\prime}\right\rangle\left\langle j_{3} \mid j_{2}^{\prime}\right\rangle \\
& =\operatorname{Tr}(D A X) \operatorname{Tr}(B) \operatorname{Tr}(C)\left\langle i_{1} \mid i_{1}^{\prime}\right\rangle .
\end{aligned}
$$

(23). If $(\pi, \sigma)=((23),(123))$, then

$$
\begin{aligned}
& \sum_{i_{2}, i_{3}, j_{1}, j_{2}, j_{3}, i_{2}^{\prime}, i_{3}^{\prime}, j_{1}^{\prime}, j_{2}^{\prime}, j_{3}^{\prime}} A_{j_{1}, j_{2}^{\prime}} B_{i_{2}^{\prime}, i_{3}} X_{j_{3}, j_{3}^{\prime}} C_{i_{3}^{\prime}, i_{2}} D_{j_{2}, j_{1}^{\prime}}\left\langle i_{1} \mid i_{1}^{\prime}\right\rangle\left\langle i_{2} \mid i_{3}^{\prime}\right\rangle\left\langle i_{3} \mid i_{2}^{\prime}\right\rangle\left\langle j_{1} \mid j_{2}^{\prime}\right\rangle\left\langle j_{2} \mid j_{3}^{\prime}\right\rangle\left\langle j_{3} \mid j_{1}^{\prime}\right\rangle \\
& =\operatorname{Tr}(A) \operatorname{Tr}(D X) \operatorname{Tr}(B) \operatorname{Tr}(C)\left\langle i_{1} \mid i_{1}^{\prime}\right\rangle .
\end{aligned}
$$

(24). If $(\pi, \sigma)=((23),(132))$, then

$$
\begin{aligned}
& \sum_{i_{2}, i_{3}, j_{1}, j_{2}, j_{3}, i_{2}^{\prime}, i_{3}^{\prime}, j_{1}^{\prime}, j_{2}^{\prime}, j_{3}^{\prime}} A_{j_{1}, j_{2}^{\prime}} B_{i_{2}^{\prime}, i_{3}} X_{j_{3}, j_{3}^{\prime}} C_{i_{3}^{\prime}, i_{2}} D_{j_{2}, j_{1}^{\prime}}\left\langle i_{1} \mid i_{1}^{\prime}\right\rangle\left\langle i_{2} \mid i_{3}^{\prime}\right\rangle\left\langle i_{3} \mid i_{2}^{\prime}\right\rangle\left\langle j_{1} \mid j_{3}^{\prime}\right\rangle\left\langle j_{2} \mid j_{1}^{\prime}\right\rangle\left\langle j_{3} \mid j_{2}^{\prime}\right\rangle \\
& =\operatorname{Tr}(D) \operatorname{Tr}(A X) \operatorname{Tr}(B) \operatorname{Tr}(C)\left\langle i_{1} \mid i_{1}^{\prime}\right\rangle .
\end{aligned}
$$

(25). If $(\pi, \sigma)=((123),(1))$, then

$$
\begin{aligned}
& \sum_{i_{2}, i_{3}, j_{1}, j_{2}, j_{3}, i_{2}^{\prime}, i_{3}^{\prime}, j_{1}^{\prime}, j_{2}^{\prime}, j_{3}^{\prime}} A_{j_{1}, j_{2}^{\prime}} B_{i_{2}^{\prime}, i_{3}} X_{j_{3}, j_{3}^{\prime}} C_{i_{3}^{\prime}, i_{2}} D_{j_{2}, j_{1}^{\prime}}\left\langle i_{1} \mid i_{2}^{\prime}\right\rangle\left\langle i_{2} \mid i_{3}^{\prime}\right\rangle\left\langle i_{3} \mid i_{1}^{\prime}\right\rangle\left\langle j_{1} \mid j_{1}^{\prime}\right\rangle\left\langle j_{2} \mid j_{2}^{\prime}\right\rangle\left\langle j_{3} \mid j_{3}^{\prime}\right\rangle \\
& =\operatorname{Tr}(A D) \operatorname{Tr}(X) \operatorname{Tr}(C)\left\langle i_{1}|B| i_{1}^{\prime}\right\rangle .
\end{aligned}
$$

(26). If $(\pi, \sigma)=((123),(12))$, then

$$
\begin{aligned}
& \sum_{i_{2}, i_{3}, j_{1}, j_{2}, j_{3}, i_{2}^{\prime}, i_{3}^{\prime}, j_{1}^{\prime}, j_{2}^{\prime}, j_{3}^{\prime}} A_{j_{1}, j_{2}^{\prime}} B_{i_{2}^{\prime}, i_{3}} X_{j_{3}, j_{3}^{\prime}} C_{i_{3}^{\prime}, i_{2}} D_{j_{2}, j_{1}^{\prime}}\left\langle i_{1} \mid i_{2}^{\prime}\right\rangle\left\langle i_{2} \mid i_{3}^{\prime}\right\rangle\left\langle i_{3} \mid i_{1}^{\prime}\right\rangle\left\langle j_{1} \mid j_{2}^{\prime}\right\rangle\left\langle j_{2} \mid j_{1}^{\prime}\right\rangle\left\langle j_{3} \mid j_{3}^{\prime}\right\rangle \\
& =\operatorname{Tr}(A) \operatorname{Tr}(D) \operatorname{Tr}(X) \operatorname{Tr}(C)\left\langle i_{1}|B| i_{1}^{\prime}\right\rangle .
\end{aligned}
$$

(27). If $(\pi, \sigma)=((123),(13))$, then

$$
\begin{aligned}
& \sum_{i_{2}, i_{3}, j_{1}, j_{2}, j_{3}, i_{2}^{\prime}, i_{3}^{\prime}, j_{1}^{\prime}, j_{2}^{\prime}, j_{3}^{\prime}} A_{j_{1}, j_{2}^{\prime}} B_{i_{2}^{\prime}, i_{3}} X_{j_{3}, j_{3}^{\prime}} C_{i_{3}^{\prime}, i_{2}} D_{j_{2}, j_{1}^{\prime}}\left\langle i_{1} \mid i_{2}^{\prime}\right\rangle\left\langle i_{2} \mid i_{3}^{\prime}\right\rangle\left\langle i_{3} \mid i_{1}^{\prime}\right\rangle\left\langle j_{1} \mid j_{3}^{\prime}\right\rangle\left\langle j_{2} \mid j_{2}^{\prime}\right\rangle\left\langle j_{3} \mid j_{1}^{\prime}\right\rangle \\
& =\operatorname{Tr}(A D X) \operatorname{Tr}(C)\left\langle i_{1}|B| i_{1}^{\prime}\right\rangle .
\end{aligned}
$$

(28). If $(\pi, \sigma)=((123),(23))$, then

$$
\begin{aligned}
& \sum_{i_{2}, i_{3}, j_{1}, j_{2}, j_{3}, i_{2}^{\prime}, i_{3}^{\prime}, j_{1}^{\prime}, j_{2}^{\prime}, j_{3}^{\prime}} A_{j_{1}, j_{2}^{\prime}} B_{i_{2}^{\prime}, i_{3}} X_{j_{3}, j_{3}^{\prime}} C_{i_{3}^{\prime}, i_{2}} D_{j_{2}, j_{1}^{\prime}}\left\langle i_{1} \mid i_{2}^{\prime}\right\rangle\left\langle i_{2} \mid i_{3}^{\prime}\right\rangle\left\langle i_{3} \mid i_{1}^{\prime}\right\rangle\left\langle j_{1} \mid j_{1}^{\prime}\right\rangle\left\langle j_{2} \mid j_{3}^{\prime}\right\rangle\left\langle j_{3} \mid j_{2}^{\prime}\right\rangle \\
& =\operatorname{Tr}(D A X) \operatorname{Tr}(C)\left\langle i_{1}|B| i_{1}^{\prime}\right\rangle .
\end{aligned}
$$


(29). If $(\pi, \sigma)=((123),(123))$, then

$$
\begin{aligned}
& \sum_{i_{2}, i_{3}, j_{1}, j_{2}, j_{3}, i_{2}^{\prime}, i_{3}^{\prime}, j_{1}^{\prime}, j_{2}^{\prime}, j_{3}^{\prime}} A_{j_{1}, j_{2}^{\prime}} B_{i_{2}^{\prime}, i_{3}} X_{j_{3}, j_{3}^{\prime}} C_{i_{3}^{\prime}, i_{2}} D_{j_{2}, j_{1}^{\prime}}\left\langle i_{1} \mid i_{2}^{\prime}\right\rangle\left\langle i_{2} \mid i_{3}^{\prime}\right\rangle\left\langle i_{3} \mid i_{1}^{\prime}\right\rangle\left\langle j_{1} \mid j_{2}^{\prime}\right\rangle\left\langle j_{2} \mid j_{3}^{\prime}\right\rangle\left\langle j_{3} \mid j_{1}^{\prime}\right\rangle \\
& =\operatorname{Tr}(A) \operatorname{Tr}(D X) \operatorname{Tr}(C)\left\langle i_{1}|B| i_{1}^{\prime}\right\rangle .
\end{aligned}
$$

(30). If $(\pi, \sigma)=((123),(132))$, then

$$
\begin{aligned}
& \sum_{i_{2}, i_{3}, j_{1}, j_{2}, j_{3}, i_{2}^{\prime}, i_{3}^{\prime}, i_{1}^{\prime}, j_{2}^{\prime}, j_{3}^{\prime}} A_{j_{1}, j_{2}^{\prime}} B_{i_{2}^{\prime}, i_{3}} X_{j_{3}, j_{3}^{\prime}} C_{i_{3}^{\prime}, i_{2}} D_{j_{2}, j_{1}^{\prime}}\left\langle i_{1} \mid i_{2}^{\prime}\right\rangle\left\langle i_{2} \mid i_{3}^{\prime}\right\rangle\left\langle i_{3} \mid i_{1}^{\prime}\right\rangle\left\langle j_{1} \mid j_{3}^{\prime}\right\rangle\left\langle j_{2} \mid j_{1}^{\prime}\right\rangle\left\langle j_{3} \mid j_{2}^{\prime}\right\rangle \\
& =\operatorname{Tr}(D) \operatorname{Tr}(A X) \operatorname{Tr}(C)\left\langle i_{1}|B| i_{1}^{\prime}\right\rangle .
\end{aligned}
$$

(31). If $(\pi, \sigma)=((132),(1))$, then

$$
\begin{aligned}
& \sum_{i_{2}, i_{3}, j_{1}, j_{2}, j_{3}, i_{2}^{\prime}, i_{3}^{\prime}, j_{1}^{\prime}, j_{2}^{\prime}, j_{3}^{\prime}} A_{j_{1}, j_{2}^{\prime}} B_{i_{2}^{\prime}, i_{3}} X_{j_{3}, j_{3}^{\prime}} C_{i_{3}^{\prime}, i_{2}} D_{j_{2}, j_{1}^{\prime}}\left\langle i_{1} \mid i_{3}^{\prime}\right\rangle\left\langle i_{2} \mid i_{1}^{\prime}\right\rangle\left\langle i_{3} \mid i_{2}^{\prime}\right\rangle\left\langle j_{1} \mid j_{1}^{\prime}\right\rangle\left\langle j_{2} \mid j_{2}^{\prime}\right\rangle\left\langle j_{3} \mid j_{3}^{\prime}\right\rangle \\
& =\operatorname{Tr}(A D) \operatorname{Tr}(X) \operatorname{Tr}(B)\left\langle i_{1}|C| i_{1}^{\prime}\right\rangle .
\end{aligned}
$$

(32). If $(\pi, \sigma)=((132),(12))$, then

$$
\begin{aligned}
& \sum_{i_{2}, i_{3}, j_{1}, j_{2}, j_{3}, i_{2}^{\prime}, i_{3}^{\prime}, j_{1}^{\prime}, j_{2}^{\prime}, j_{3}^{\prime}} A_{j_{1}, j_{2}^{\prime}} B_{i_{2}^{\prime}, i_{3}} X_{j_{3}, j_{3}^{\prime}} C_{i_{3}^{\prime}, i_{2}} D_{j_{2}, j_{1}^{\prime}}\left\langle i_{1} \mid i_{3}^{\prime}\right\rangle\left\langle i_{2} \mid i_{1}^{\prime}\right\rangle\left\langle i_{3} \mid i_{2}^{\prime}\right\rangle\left\langle j_{1} \mid j_{2}^{\prime}\right\rangle\left\langle j_{2} \mid j_{1}^{\prime}\right\rangle\left\langle j_{3} \mid j_{3}^{\prime}\right\rangle \\
& =\operatorname{Tr}(A) \operatorname{Tr}(D) \operatorname{Tr}(X) \operatorname{Tr}(B)\left\langle i_{1}|C| i_{1}^{\prime}\right\rangle .
\end{aligned}
$$

(33). If $(\pi, \sigma)=((132),(13))$, then

$$
\begin{aligned}
& \sum_{i_{2}, i_{3}, j_{1}, j_{2}, j_{3}, i_{2}^{\prime}, i_{3}^{\prime}, j_{1}^{\prime}, j_{2}^{\prime}, j_{3}^{\prime}} A_{j_{1}, j_{2}^{\prime}} B_{i_{2}^{\prime}, i_{3}} X_{j_{3}, j_{3}^{\prime}} C_{i_{3}^{\prime}, i_{2}} D_{j_{2}, j_{1}^{\prime}}\left\langle i_{1} \mid i_{3}^{\prime}\right\rangle\left\langle i_{2} \mid i_{1}^{\prime}\right\rangle\left\langle i_{3} \mid i_{2}^{\prime}\right\rangle\left\langle j_{1} \mid j_{3}^{\prime}\right\rangle\left\langle j_{2} \mid j_{2}^{\prime}\right\rangle\left\langle j_{3} \mid j_{1}^{\prime}\right\rangle \\
& =\operatorname{Tr}(A D X) \operatorname{Tr}(B)\left\langle i_{1}|C| i_{1}^{\prime}\right\rangle .
\end{aligned}
$$

(34). If $(\pi, \sigma)=((132),(23))$, then

$$
\begin{aligned}
& \sum_{i_{2}, i_{3}, j_{1}, j_{2}, j_{3}, i_{2}^{\prime}, i_{3}, j_{1}^{\prime}, j_{2}^{\prime}, j_{3}^{\prime}} A_{j_{1}, j_{2}^{\prime}} B_{i_{2}^{\prime}, i_{3}} X_{j_{3}, j_{3}^{\prime}} C_{i_{3}^{\prime}, i_{2}} D_{j_{2}, j_{1}^{\prime}}\left\langle i_{1} \mid i_{3}^{\prime}\right\rangle\left\langle i_{2} \mid i_{1}^{\prime}\right\rangle\left\langle i_{3} \mid i_{2}^{\prime}\right\rangle\left\langle j_{1} \mid j_{1}^{\prime}\right\rangle\left\langle j_{2} \mid j_{3}^{\prime}\right\rangle\left\langle j_{3} \mid j_{2}^{\prime}\right\rangle \\
& =\operatorname{Tr}(D A X) \operatorname{Tr}(B)\left\langle i_{1}|C| i_{1}^{\prime}\right\rangle .
\end{aligned}
$$

(35). If $(\pi, \sigma)=((132),(123))$, then

$$
\begin{aligned}
& \sum_{i_{2}, i_{3}, j_{1}, j_{2}, j_{3}, i_{2}^{\prime}, i_{3}^{\prime}, j_{1}^{\prime}, j_{2}^{\prime}, j_{3}^{\prime}} A_{j_{1}, j_{2}^{\prime}} B_{i_{2}^{\prime}, i_{3}} X_{j_{3}, j_{3}^{\prime}} C_{i_{3}^{\prime}, i_{2}} D_{j_{2}, j_{1}^{\prime}}\left\langle i_{1} \mid i_{3}^{\prime}\right\rangle\left\langle i_{2} \mid i_{1}^{\prime}\right\rangle\left\langle i_{3} \mid i_{2}^{\prime}\right\rangle\left\langle j_{1} \mid j_{2}^{\prime}\right\rangle\left\langle j_{2} \mid j_{3}^{\prime}\right\rangle\left\langle j_{3} \mid j_{1}^{\prime}\right\rangle \\
& =\operatorname{Tr}(A) \operatorname{Tr}(D X) \operatorname{Tr}(B)\left\langle i_{1}|C| i_{1}^{\prime}\right\rangle .
\end{aligned}
$$

(36). If $(\pi, \sigma)=((132),(132))$, then

$$
\begin{aligned}
& \sum_{i_{2}, i_{3}, j_{1}, j_{2}, j_{3}, i_{2}^{\prime}, i_{3}^{\prime}, j_{1}^{\prime}, j_{2}^{\prime}, j_{3}^{\prime}} A_{j_{1}, j_{2}^{\prime}} B_{i_{2}^{\prime}, i_{3}} X_{j_{3}, j_{3}^{\prime}} C_{i_{3}^{\prime}, i_{2}} D_{j_{2}, j_{1}^{\prime}}\left\langle i_{1} \mid i_{3}^{\prime}\right\rangle\left\langle i_{2} \mid i_{1}^{\prime}\right\rangle\left\langle i_{3} \mid i_{2}^{\prime}\right\rangle\left\langle j_{1} \mid j_{3}^{\prime}\right\rangle\left\langle j_{2} \mid j_{1}^{\prime}\right\rangle\left\langle j_{3} \mid j_{2}^{\prime}\right\rangle \\
& =\operatorname{Tr}(D) \operatorname{Tr}(A X) \operatorname{Tr}(B)\left\langle i_{1}|C| i_{1}^{\prime}\right\rangle .
\end{aligned}
$$

Combing the 36 cases together gives the desired conclusion:

$$
\int U A U^{\dagger} B U X U^{\dagger} C U D U^{\dagger} \mathrm{d} \mu(U)=\mu_{1} \cdot \mathbb{1}_{d}+\mu_{2} \cdot B C+\mu_{3} \cdot C B+\mu_{4} \cdot B+\mu_{5} \cdot C,
$$


where the coefficients $\mu_{j}(j=1, \ldots, 5)$ are given below:

$$
\begin{aligned}
\mu_{1}:= & \operatorname{Wg}(1,1,1) \operatorname{Tr}(A D) \operatorname{Tr}(X) \operatorname{Tr}(B C)+\mathrm{Wg}(2,1) \operatorname{Tr}(A) \operatorname{Tr}(D) \operatorname{Tr}(X) \operatorname{Tr}(B C) \\
& +\mathrm{Wg}(2,1) \operatorname{Tr}(A D X) \operatorname{Tr}(B C)+\mathrm{Wg}(2,1) \operatorname{Tr}(D A X) \operatorname{Tr}(B C) \\
& +\mathrm{Wg}(3) \operatorname{Tr}(A) \operatorname{Tr}(D X) \operatorname{Tr}(B C)+\mathrm{Wg}(3) \operatorname{Tr}(D) \operatorname{Tr}(A X) \operatorname{Tr}(B C) \\
& +\mathrm{Wg}(2,1) \operatorname{Tr}(A D) \operatorname{Tr}(X) \operatorname{Tr}(B) \operatorname{Tr}(C)+\mathrm{Wg}(3) \operatorname{Tr}(A) \operatorname{Tr}(D) \operatorname{Tr}(X) \operatorname{Tr}(B) \operatorname{Tr}(C) \\
& +\mathrm{Wg}(3) \operatorname{Tr}(A D X) \operatorname{Tr}(B) \operatorname{Tr}(C)+\mathrm{Wg}(1,1,1) \operatorname{Tr}(D A X) \operatorname{Tr}(B) \operatorname{Tr}(C) \\
& +\mathrm{Wg}(2,1) \operatorname{Tr}(A) \operatorname{Tr}(D X) \operatorname{Tr}(B) \operatorname{Tr}(C)+\mathrm{Wg}(2,1) \operatorname{Tr}(D) \operatorname{Tr}(A X) \operatorname{Tr}(B) \operatorname{Tr}(C), \\
\mu_{2}:= & \mathrm{Wg}(2,1) \operatorname{Tr}(A D) \operatorname{Tr}(X)+\mathrm{Wg}(1,1,1) \operatorname{Tr}(A) \operatorname{Tr}(D) \operatorname{Tr}(X)+\mathrm{Wg}(3) \operatorname{Tr}(A D X) \\
& +\mathrm{Wg}(3) \operatorname{Tr}(D A X)+\mathrm{Wg}(2,1) \operatorname{Tr}(A) \operatorname{Tr}(D X)+\mathrm{Wg}(2,1) \operatorname{Tr}(D) \operatorname{Tr}(A X), \\
\mu_{3}:= & \mathrm{Wg}(2,1) \operatorname{Tr}(A D) \operatorname{Tr}(X)+\mathrm{Wg}(3) \operatorname{Tr}(A) \operatorname{Tr}(D) \operatorname{Tr}(X)+\mathrm{Wg}(1,1,1) \operatorname{Tr}(A D X) \\
& +\mathrm{Wg}(3) \operatorname{Tr}(D A X)+\mathrm{Wg}(2,1) \operatorname{Tr}(A) \operatorname{Tr}(D X)+\mathrm{Wg}(2,1) \operatorname{Tr}(D) \operatorname{Tr}(A X), \\
\mu_{4}:= & \mathrm{Wg}(3) \operatorname{Tr}(A D) \operatorname{Tr}(X) \operatorname{Tr}(C)+\mathrm{Wg}(2,1) \operatorname{Tr}(A) \operatorname{Tr}(D) \operatorname{Tr}(X) \operatorname{Tr}(C) \\
& +W g(2,1) \operatorname{Tr}(A D X) \operatorname{Tr}(C)+\mathrm{Wg}(2,1) \operatorname{Tr}(D A X) \operatorname{Tr}(C) \\
& +W g(1,1,1) \operatorname{Tr}(A) \operatorname{Tr}(D X) \operatorname{Tr}(C)+\mathrm{Wg}(3) \operatorname{Tr}(D) \operatorname{Tr}(A X) \operatorname{Tr}(C), \\
\mu_{5}= & W g(3) \operatorname{Tr}(A D) \operatorname{Tr}(X) \operatorname{Tr}(B)+\mathrm{Wg}(2,1) \operatorname{Tr}(A) \operatorname{Tr}(D) \operatorname{Tr}(X) \operatorname{Tr}(B) \\
& +W g(2,1) \operatorname{Tr}(A D X) \operatorname{Tr}(B)+\mathrm{Wg}(2,1) \operatorname{Tr}(D A X) \operatorname{Tr}(B) \\
& +W g(3) \operatorname{Tr}(A) \operatorname{Tr}(D X) \operatorname{Tr}(B)+\mathrm{Wg}(1,1,1) \operatorname{Tr}(D) \operatorname{Tr}(A X) \operatorname{Tr}(B) .
\end{aligned}
$$

We are done.

Remark 4.1. $\mathrm{Wg}:=\frac{1}{(k !)^{2}} \sum_{\lambda \vdash k} \frac{\operatorname{dim}\left(\mathbf{P}_{\lambda}\right)^{2}}{\operatorname{dim}\left(\mathbf{Q}_{\lambda}\right)} \chi_{\lambda}$ is called the Weingarten function [4]. In particular, for $\lambda \vdash 3$, we have:

$$
\begin{aligned}
\mathrm{Wg}(1,1,1) & =\frac{d^{2}-2}{d\left(d^{2}-1\right)\left(d^{2}-4\right)}=\left(d-\frac{2}{d}\right) \cdot \frac{1}{N_{d}} \\
\mathrm{Wg}(2,1) & =-\frac{1}{\left(d^{2}-1\right)\left(d^{2}-4\right)}=(-1) \cdot \frac{1}{N_{d}} \\
\mathrm{Wg}(3) & =\frac{2}{d\left(d^{2}-1\right)\left(d^{2}-4\right)}=\frac{2}{d} \cdot \frac{1}{N_{d}}
\end{aligned}
$$


where $N_{d}=\left(d^{2}-1\right)\left(d^{2}-4\right)$. With these coefficients, we then have

$$
\begin{aligned}
N_{d} \mu_{1}:= & \left(d-\frac{2}{d}\right) \operatorname{Tr}(A D) \operatorname{Tr}(X) \operatorname{Tr}(B C)+\left(d-\frac{2}{d}\right) \operatorname{Tr}(D A X) \operatorname{Tr}(B) \operatorname{Tr}(C)+\frac{2}{d} \operatorname{Tr}(A) \operatorname{Tr}(D X) \operatorname{Tr}(B C) \\
& +\frac{2}{d} \operatorname{Tr}(D) \operatorname{Tr}(A X) \operatorname{Tr}(B C)+\frac{2}{d} \operatorname{Tr}(A) \operatorname{Tr}(D) \operatorname{Tr}(X) \operatorname{Tr}(B) \operatorname{Tr}(C)+\frac{2}{d} \operatorname{Tr}(A D X) \operatorname{Tr}(B) \operatorname{Tr}(C) \\
& -\operatorname{Tr}(A) \operatorname{Tr}(D) \operatorname{Tr}(X) \operatorname{Tr}(B C)-\operatorname{Tr}(A D X) \operatorname{Tr}(B C)-\operatorname{Tr}(D A X) \operatorname{Tr}(B C) \\
& -\operatorname{Tr}(A D) \operatorname{Tr}(X) \operatorname{Tr}(B) \operatorname{Tr}(C)-\operatorname{Tr}(A) \operatorname{Tr}(D X) \operatorname{Tr}(B) \operatorname{Tr}(C)-\operatorname{Tr}(D) \operatorname{Tr}(A X) \operatorname{Tr}(B) \operatorname{Tr}(C(4.85) \\
N_{d} \mu_{2}:= & \left(d-\frac{2}{d}\right) \operatorname{Tr}(A) \operatorname{Tr}(D) \operatorname{Tr}(X)+\frac{2}{d} \operatorname{Tr}(A D X)+\frac{2}{d} \operatorname{Tr}(D A X) \\
& -\operatorname{Tr}(A D) \operatorname{Tr}(X)-\operatorname{Tr}(A) \operatorname{Tr}(D X)-\operatorname{Tr}(D) \operatorname{Tr}(A X), \\
N_{d} \mu_{3}:= & \frac{2}{d} \operatorname{Tr}(D A X)+\frac{2}{d} \operatorname{Tr}(A) \operatorname{Tr}(D) \operatorname{Tr}(X)+\left(d-\frac{2}{d}\right) \operatorname{Tr}(A D X) \\
& -\operatorname{Tr}(A D) \operatorname{Tr}(X)-\operatorname{Tr}(A) \operatorname{Tr}(D X)-\operatorname{Tr}(D) \operatorname{Tr}(A X), \\
N_{d} \mu_{4}:= & \left(d-\frac{2}{d}\right) \operatorname{Tr}(A) \operatorname{Tr}(D X) \operatorname{Tr}(C)+\frac{2}{d} \operatorname{Tr}(A D) \operatorname{Tr}(X) \operatorname{Tr}(C)+\frac{2}{d} \operatorname{Tr}(D) \operatorname{Tr}(A X) \operatorname{Tr}(C) \\
& -\operatorname{Tr}(A) \operatorname{Tr}(D) \operatorname{Tr}(X) \operatorname{Tr}(C)-\operatorname{Tr}(A D X) \operatorname{Tr}(C)-\operatorname{Tr}(D A X) \operatorname{Tr}(C) \\
N_{d} \mu_{5}:= & \frac{2}{d} \operatorname{Tr}(A D) \operatorname{Tr}(X) \operatorname{Tr}(B)+\frac{2}{d} \operatorname{Tr}(A) \operatorname{Tr}(D X) \operatorname{Tr}(B)+\left(d-\frac{2}{d}\right) \operatorname{Tr}(D) \operatorname{Tr}(A X) \operatorname{Tr}(B) \\
& -\operatorname{Tr}(A) \operatorname{Tr}(D) \operatorname{Tr}(X) \operatorname{Tr}(B)-\operatorname{Tr}(A D X) \operatorname{Tr}(B)-\operatorname{Tr}(D A X) \operatorname{Tr}(B) .
\end{aligned}
$$

\section{B. The proof of Theorem 2.2}

By Lemma 2.1,

$$
\int U^{\dagger} M_{i j} U T U^{\dagger} M_{j l} U T U^{\dagger} M_{l i} U \mathrm{~d} \mu(U)=f(i, j, l) \cdot \mathbb{1}_{d}+g(i, j, l) \cdot T+h(i, j, l) \cdot T^{2},
$$

where

$$
\begin{aligned}
& f(i, j, l)=\mathrm{Wg}(1,1,1) d_{A}^{2} \delta_{j l} \operatorname{Tr}\left(T^{2}\right)+\mathrm{Wg}(2,1) d_{A}^{3} \delta_{i j} \delta_{j l} \delta_{l i} \operatorname{Tr}\left(T^{2}\right)+\mathrm{Wg}(2,1) d_{A} \delta_{i j} \delta_{j l} \delta_{l i} \operatorname{Tr}\left(T^{2}\right) \\
& +\mathrm{Wg}(2,1) d_{A} \operatorname{Tr}\left(T^{2}\right)+\mathrm{Wg}(3) d_{A}^{2} \delta_{i j} \operatorname{Tr}\left(T^{2}\right)+\mathrm{Wg}(3) d_{A}^{2} \delta_{i l} \operatorname{Tr}\left(T^{2}\right) \\
& +\mathrm{Wg}(2,1) d_{A}^{2} \delta_{j l} \operatorname{Tr}(T)^{2}+\mathrm{Wg}(3) d_{A}^{3} \delta_{i j} \delta_{j l} \delta_{l i} \operatorname{Tr}(T)^{2}+\mathrm{Wg}(3) d_{A} \delta_{i j} \delta_{j l} \delta_{l i} \operatorname{Tr}(T)^{2} \\
& +\mathrm{Wg}(1,1,1) d_{A} \operatorname{Tr}(T)^{2}+\mathrm{Wg}(2,1) d_{A}^{2} \delta_{i j} \operatorname{Tr}(T)^{2}+\mathrm{Wg}(2,1) d_{A}^{2} \delta_{i l} \operatorname{Tr}(T)^{2} \text {, } \\
& g(i, j, l)=\mathrm{Wg}(3) d_{A}^{2} \delta_{j l} \operatorname{Tr}(T)+\mathrm{Wg}(2,1) d_{A}^{3} \delta_{i j} \delta_{j l} \delta_{l i} \operatorname{Tr}(T)+\mathrm{Wg}(2,1) d_{A} \delta_{i j} \delta_{j l} \delta_{l i} \operatorname{Tr}(T) \\
& +\mathrm{Wg}(2,1) d_{A} \operatorname{Tr}(T)+\mathrm{Wg}(1,1,1) d_{A}^{2} \delta_{i j} \operatorname{Tr}(T)+\mathrm{Wg}(3) d_{A}^{2} \delta_{i l} \operatorname{Tr}(T) \\
& +\mathrm{Wg}(3) d_{A}^{2} \delta_{j l} \operatorname{Tr}(T)+\mathrm{Wg}(2,1) d_{A}^{3} \delta_{i j} \delta_{j l} \delta_{l i} \operatorname{Tr}(T)+\mathrm{Wg}(2,1) d_{A} \delta_{i j} \delta_{j l} \delta_{l i} \operatorname{Tr}(T) \\
& +\mathrm{Wg}(2,1) d_{A} \operatorname{Tr}(T)+\mathrm{Wg}(3) d_{A}^{2} \delta_{i j} \operatorname{Tr}(T)+\mathrm{Wg}(1,1,1) d_{A}^{2} \delta_{i l} \operatorname{Tr}(T), \\
& h(i, j, l)=\operatorname{Wg}(2,1) d_{A}^{2} \delta_{j l}+\operatorname{Wg}(1,1,1) d_{A}^{3} \delta_{i j} \delta_{j l} \delta_{l i}+\operatorname{Wg}(3) d_{A} \delta_{i j} \delta_{j l} \delta_{l i} \\
& +\mathrm{Wg}(3) d_{A}+\mathrm{Wg}(2,1) d_{A}^{2} \delta_{i j}+\mathrm{Wg}(2,1) d_{A}^{2} \delta_{i l}, \\
& +\mathrm{Wg}(2,1) d_{A}^{2} \delta_{j l}+\mathrm{Wg}(3) d_{A}^{3} \delta_{i j} \delta_{j l} \delta_{l i}+\mathrm{Wg}(1,1,1) d_{A} \delta_{i j} \delta_{j l} \delta_{l i} \\
& +\mathrm{Wg}(3) d_{A}+\mathrm{Wg}(2,1) d_{A}^{2} \delta_{i j}+\mathrm{Wg}(2,1) d_{A}^{2} \delta_{i l} \text {. }
\end{aligned}
$$


Note that the meaning of the notation $\operatorname{Wg}(*)$ can be found in the Appendix. Thus for $f:=\sum_{i, j, l=1}^{d_{B}} f(i, j, l)$, $g:=\sum_{i, j, l=1}^{d_{B}} g(i, j, l)$, and $h:=\sum_{i, j, l=1}^{d_{B}} h(i, j, l)$, we have

$$
\begin{aligned}
f= & \left([\mathrm{Wg}(1,1,1)+2 \mathrm{Wg}(3)] d^{2}+\mathrm{Wg}(2,1)\left(d d_{A}^{2}+d+d d_{B}^{2}\right)\right) \operatorname{Tr}\left(T^{2}\right) \\
& +\left(3 \mathrm{Wg}(2,1) d^{2}+\mathrm{Wg}(3)\left(d d_{A}^{2}+d\right)+\mathrm{Wg}(1,1,1) d d_{B}^{2}\right) \operatorname{Tr}(T)^{2}, \\
g= & \left([4 \mathrm{Wg}(3)+2 \mathrm{Wg}(1,1,1)] d^{2}+2 \mathrm{Wg}(2,1)\left(d d_{A}^{2}+d+d d_{B}^{2}\right)\right) \operatorname{Tr}(T), \\
h= & 6 \mathrm{Wg}(2,1) d^{2}+[\mathrm{Wg}(1,1,1)+\mathrm{Wg}(3)] d d_{A}^{2}+[\mathrm{Wg}(1,1,1)+\mathrm{Wg}(3)] d+2 \mathrm{Wg}(3) d d_{B}^{2} .
\end{aligned}
$$

Hence, for $T=\mathbb{1}_{A} \otimes \mathbb{1}_{B} / d_{B}-\rho_{A B}, \operatorname{Tr}(T)=d_{A}-1$ and $\operatorname{Tr}\left(T^{2}\right)=\frac{d_{A}-2}{d_{B}}+\operatorname{Tr}\left(\rho_{A B}^{2}\right)$, then

$$
\begin{aligned}
f= & \frac{d\left(d^{2}-d_{A}^{2}-d_{B}^{2}+1\right)}{\left(d^{2}-1\right)\left(d^{2}-4\right)} \operatorname{Tr}\left(T^{2}\right)+\frac{d^{2}\left(d_{B}^{2}-3\right)+2\left(d_{A}^{2}-d_{B}^{2}+1\right)}{\left(d^{2}-1\right)\left(d^{2}-4\right)} \operatorname{Tr}(T)^{2}, \\
= & \frac{d_{A}\left(d_{A}^{2}-1\right)\left(d_{B}^{2}-1\right)}{\left(d^{2}-1\right)\left(d^{2}-4\right)}\left(d_{A}+d_{B} \operatorname{Tr}\left(\rho_{A B}^{2}\right)-2\right) \\
& +\frac{\left(d^{2}-2 d_{A}^{2}-2\right)\left(d_{B}^{2}-1\right)}{\left(d^{2}-1\right)\left(d^{2}-4\right)}\left(d_{A}-1\right)^{2}, \\
g= & \frac{2 d\left(d^{2}-d_{A}^{2}-d_{B}^{2}+1\right)}{\left(d^{2}-1\right)\left(d^{2}-4\right)} \operatorname{Tr}(T)=\frac{2 d\left(d_{A}-1\right)\left(d_{A}^{2}-1\right)\left(d_{B}^{2}-1\right)}{\left(d^{2}-1\right)\left(d^{2}-4\right)}, \\
h= & \frac{d^{2}\left(d_{A}^{2}-5\right)+4 d_{B}^{2}}{\left(d^{2}-1\right)\left(d^{2}-4\right)}=\frac{\left(d_{A}^{2}-1\right)\left(d_{A}^{2}-4\right) d_{B}^{2}}{\left(d^{2}-1\right)\left(d^{2}-4\right)} .
\end{aligned}
$$

Therefore,

$$
\int U^{\dagger}\left[\Gamma\left(U T U^{\dagger}\right)\right]^{2} U \mathrm{~d} \mu(U)=f \cdot \mathbb{1}_{d}+g \cdot T+h \cdot T^{2}
$$

implying that

$$
\begin{aligned}
a_{2} & =f+g \cdot \operatorname{Tr}\left(\rho_{A B} T\right)+h \cdot \operatorname{Tr}\left(\rho_{A B} T^{2}\right) \\
& =f+g \cdot\left(\frac{1}{d_{B}}-\operatorname{Tr}\left(\rho_{A B}^{2}\right)\right)+h \cdot\left(\frac{1}{d_{B}^{2}}-\frac{2}{d_{B}} \operatorname{Tr}\left(\rho_{A B}^{2}\right)+\operatorname{Tr}\left(\rho_{A B}^{3}\right)\right) \\
& =\left(f+g \frac{1}{d_{B}}+h \frac{1}{d_{B}^{2}}\right)-\left(g+h \frac{2}{d_{B}}\right) \operatorname{Tr}\left(\rho_{A B}^{2}\right)+h \operatorname{Tr}\left(\rho_{A B}^{3}\right) .
\end{aligned}
$$

We make further analysis of the term $a_{n}$ although we have already known the fact that $\lim _{n \rightarrow \infty} a_{n}=0$. Let $\varphi_{\rho}(X)=\operatorname{Tr}(\rho X)$. Apparently, $\varphi_{\rho}$ is a positive unital linear mapping (in fact, it is a positive unital linear functional from the set of $n \times n$ Hermitian matrices to $\mathbb{R}$ ). It is easily seen that $f(x)=x^{n}$ is a convex function from $\mathbb{R}$ to $\mathbb{R}$. By using [10, Theorem 4.15, pp147], we see that

$$
\begin{aligned}
a_{n} & =\varphi_{\rho}\left(\int\left[U^{\dagger} \Gamma\left(U T U^{\dagger}\right) U\right]^{n} \mathrm{~d} \mu(U)\right)=\varphi_{\rho}\left(\int f\left(U^{\dagger} \Gamma\left(U T U^{\dagger}\right) U\right) \mathrm{d} \mu(U)\right) \\
& =\int \mathrm{d} \mu(U)\left(\varphi_{\rho} \circ f\right)\left(U^{\dagger} \Gamma\left(U T U^{\dagger}\right) U\right) \geqslant \int \mathrm{d} \mu(U) f\left[\varphi_{\rho}\left(U^{\dagger} \Gamma\left(U T U^{\dagger}\right) U\right)\right] \\
& \geqslant \int \mathrm{d} \mu(U)\left[\operatorname{Tr}\left(\rho U^{\dagger} \Gamma\left(U T U^{\dagger}\right) U\right)\right]^{n} \geqslant\left(\operatorname{Tr}\left(\rho \int \mathrm{d} \mu(U) U^{\dagger} \Gamma\left(U T U^{\dagger}\right) U\right)\right)^{n} .
\end{aligned}
$$


By (2.4), we have

$$
\operatorname{Tr}\left(\rho \int \mathrm{d} \mu(U) U^{\dagger} \Gamma\left(U T U^{\dagger}\right) U\right)=\frac{d_{A}-1}{d^{2}-1}\left[\left(1+d d_{B}\right)-\left(d+d_{B}\right) \operatorname{Tr}\left(\rho_{A B}^{2}\right)\right]=a_{1} .
$$

Thus

$$
a_{n} \geqslant a_{1}^{n}
$$

Then

$$
a_{1}=\frac{\left(d_{A}-1\right)\left(d_{B}-1\right)}{d+1}+\frac{\left(d_{A}-1\right)\left(d+d_{B}\right)}{d^{2}-1} S_{L}\left(\rho_{A B}\right),
$$

where $\mathrm{S}_{L}\left(\rho_{A B}\right) \in\left[0,1-\frac{1}{d}\right]$. Clearly

$$
\frac{\left(d_{A}-1\right)\left(d_{B}-1\right)}{d+1} \leqslant a_{1} \leqslant \frac{\left(d_{A}-1\right)\left(1+\frac{1}{d_{A}}\right)}{d+1}<1 .
$$

Now

$$
\int \mathrm{S}\left(\rho_{A}^{\prime}\right) \mathrm{d} \mu(U)=\sum_{n=1}^{\infty} \frac{a_{n}}{n} \geqslant \sum_{n=1}^{\infty} \frac{a_{1}^{n}}{n}=-\ln \left(1-a_{1}\right) .
$$

We see from the above lower bound, i.e., $-\ln \left(1-a_{1}\right)$, that when the purity of $\rho_{A B}$ decreases, $a_{1}$ increases. Hence such lower bound will be tighter.

\section{The proof of Proposition 3.2}

Clearly, the first inequality is easily obtained

$$
\begin{aligned}
\int I(A: B)_{\rho^{\prime}} \mathrm{d} \mu(U) & \geqslant \mathrm{S}\left(\rho_{A B} \| \int \ln \left[U^{\dagger}\left(\rho_{A}^{\prime} \otimes \rho_{B}^{\prime}\right) U\right] \mathrm{d} \mu(U)\right) \\
& =\mathrm{S}\left(\rho_{A B} \| c_{0} \cdot \mathbb{1}_{d}+c_{1} \cdot \rho_{A B}+c_{2} \cdot \rho_{A B}^{2}\right) .
\end{aligned}
$$

In order to show the second inequality, note that, for any two density matrices $\rho$ and $\sigma$,

$$
\mathrm{F}(\rho, \sigma) \geqslant \operatorname{Tr}(\sqrt{\rho} \sqrt{\sigma}) \geqslant \operatorname{Tr}(\rho \sigma) .
$$

Then, for $\rho_{A B}^{\prime}=U \rho_{A B} U^{\dagger}$, we have

$$
\mathrm{F}\left(\rho_{A B}^{\prime}, \rho_{A}^{\prime} \otimes \rho_{B}^{\prime}\right) \geqslant \operatorname{Tr}\left(\rho_{A B}^{\prime} \rho_{A}^{\prime} \otimes \rho_{B}^{\prime}\right)=\operatorname{Tr}\left(\rho_{A B} U^{+}\left(\rho_{A}^{\prime} \otimes \rho_{B}^{\prime}\right) U\right),
$$

implying that

$$
\int \mathrm{F}\left(\rho_{A B}^{\prime}, \rho_{A}^{\prime} \otimes \rho_{B}^{\prime}\right) \mathrm{d} \mu(U) \geqslant \int \operatorname{Tr}\left(\rho_{A B} U^{+}\left(\rho_{A}^{\prime} \otimes \rho_{B}^{\prime}\right) U\right) \mathrm{d} \mu(U)=c_{0}+c_{1} \operatorname{Tr}\left(\rho_{A B}^{2}\right)+c_{2} \operatorname{Tr}\left(\rho_{A B}^{3}\right) .
$$

By the concavity of fidelity, we have

$$
\int \mathrm{F}\left(\rho_{A B}^{\prime}, \rho_{A}^{\prime} \otimes \rho_{B}^{\prime}\right) \mathrm{d} \mu(U)=\int \mathrm{F}\left(\rho_{A B}, U^{\dagger}\left(\rho_{A}^{\prime} \otimes \rho_{B}^{\prime}\right) U\right) \mathrm{d} \mu(U) \leqslant \mathrm{F}\left(\rho_{A B}, c_{0} \cdot \mathbb{1}_{d}+c_{1} \cdot \rho_{A B}+c_{2} \cdot \rho_{A B}^{2}\right) \text {. }
$$

Therefore

$$
c_{0}+c_{1} \operatorname{Tr}\left(\rho_{A B}^{2}\right)+c_{2} \operatorname{Tr}\left(\rho_{A B}^{3}\right) \leqslant \int \mathrm{F}\left(\rho_{A B}^{\prime}, \rho_{A}^{\prime} \otimes \rho_{B}^{\prime}\right) \mathrm{d} \mu(U) \leqslant \mathrm{F}\left(\rho_{A B}, c_{0} \cdot \mathbb{1}_{d}+c_{1} \cdot \rho_{A B}+c_{2} \cdot \rho_{A B}^{2}\right) .
$$

This completes the proof. 


\section{The proof of Theorem 3.4}

Note that $\rho_{A B}^{\prime}=U \rho_{A B} U^{\dagger}$. We see from (3.8) that

$$
\int I(A: B)_{\rho^{\prime}} \mathrm{d} \mu(U) \geqslant \mathrm{S}\left(\rho_{A B} \| c_{0} \cdot \mathbb{1}_{d}+c_{1} \cdot \rho_{A B}+c_{2} \cdot \rho_{A B}^{2}\right) .
$$

Since $I(A: B)_{\rho^{\prime}}=\mathrm{S}\left(\rho_{A}^{\prime}\right)+\mathrm{S}\left(\rho_{B}^{\prime}\right)-\mathrm{S}\left(\rho_{A B}\right)$, it follows that

$$
\int\left(\mathrm{S}\left(\rho_{A}^{\prime}\right)+\mathrm{S}\left(\rho_{B}^{\prime}\right)-\mathrm{S}\left(\rho_{A B}\right)\right) \mathrm{d} \mu(U) \geqslant \mathrm{S}\left(\rho_{A B} \| c_{0} \cdot \mathbb{1}_{d}+c_{1} \cdot \rho_{A B}+c_{2} \cdot \rho_{A B}^{2}\right) .
$$

That is,

$$
\left\langle S_{A}+S_{B}\right\rangle \geqslant \mathrm{S}\left(\rho_{A B}\right)+\mathrm{S}\left(\rho_{A B} \| c_{0} \cdot \mathbb{1}_{d}+c_{1} \cdot \rho_{A B}+c_{2} \cdot \rho_{A B}^{2}\right) .
$$

This confirms the first inequality. Besides, by Eq. (3.4), we get

$$
\begin{aligned}
& \mathrm{S}\left(c_{0} \cdot \mathbb{1}_{d}+c_{1} \cdot \rho_{A B}+c_{2} \cdot \rho_{A B}^{2}\right)=\mathrm{S}\left(\int U^{\dagger}\left(\rho_{A}^{\prime} \otimes \rho_{B}^{\prime}\right) U \mathrm{~d} \mu(U)\right) \\
& \geqslant \int \mathrm{S}\left(U^{\dagger}\left(\rho_{A}^{\prime} \otimes \rho_{B}^{\prime}\right) U\right) \mathrm{d} \mu(U)=\int \mathrm{S}\left(\rho_{A}^{\prime}\right) \mathrm{d} \mu(U)+\int \mathrm{S}\left(\rho_{B}^{\prime}\right) \mathrm{d} \mu(U) .
\end{aligned}
$$

This confirms the second inequality. Therefore we have

$$
\mathrm{S}\left(\rho_{A B}\right)+\mathrm{S}\left(\rho_{A B} \| c_{0} \cdot \mathbb{1}_{d}+c_{1} \cdot \rho_{A B}+c_{2} \cdot \rho_{A B}^{2}\right) \leqslant \mathrm{S}\left(c_{0} \cdot \mathbb{1}_{d}+c_{1} \cdot \rho_{A B}+c_{2} \cdot \rho_{A B}^{2}\right) .
$$

This is equivalent to the following

$$
\mathrm{S}\left(\rho_{A B} \| c_{0} \cdot \mathbb{1}_{d}+c_{1} \cdot \rho_{A B}+c_{2} \cdot \rho_{A B}^{2}\right) \leqslant \mathrm{S}\left(c_{0} \cdot \mathbb{1}_{d}+c_{1} \cdot \rho_{A B}+c_{2} \cdot \rho_{A B}^{2}\right)-\mathrm{S}\left(\rho_{A B}\right) .
$$

Next we show that $\mathrm{S}\left(c_{0} \cdot \mathbb{1}_{d}+c_{1} \cdot \rho_{A B}+c_{2} \cdot \rho_{A B}^{2}\right)=\mathrm{S}\left(\rho_{A B}\right)$ if and only if $\rho_{A B}$ is maximally mixed state. Clearly if $\rho_{A B}$ is maximally mixed state, i.e., $S\left(\rho_{A B}\right)=\ln (d)$, since $S\left(c_{0} \cdot \mathbb{1}_{d}+c_{1} \cdot \rho_{A B}+c_{2} \cdot \rho_{A B}^{2}\right)-S\left(\rho_{A B}\right) \geqslant$ 0 , then $\mathrm{S}\left(c_{0} \cdot \mathbb{1}_{d}+c_{1} \cdot \rho_{A B}+c_{2} \cdot \rho_{A B}^{2}\right) \geqslant \ln (d)$, apparently $\mathrm{S}\left(c_{0} \cdot \mathbb{1}_{d}+c_{1} \cdot \rho_{A B}+c_{2} \cdot \rho_{A B}^{2}\right) \leqslant \ln (d)$, thus $\mathrm{S}\left(\rho_{A B}\right)=\mathrm{S}\left(c_{0} \cdot \mathbb{1}_{d}+c_{1} \cdot \rho_{A B}+c_{2} \cdot \rho_{A B}^{2}\right)=\ln (d)$, the maximum of von Neuman entropy. Reversely, if $\mathrm{S}\left(c_{0} \cdot \mathbb{1}_{d}+c_{1} \cdot \rho_{A B}+c_{2} \cdot \rho_{A B}^{2}\right)=\mathrm{S}\left(\rho_{A B}\right)$, then by the obtained inequality, we have

$$
\mathrm{S}\left(\rho_{A B} \| c_{0} \cdot \mathbb{1}_{d}+c_{1} \cdot \rho_{A B}+c_{2} \cdot \rho_{A B}^{2}\right)=0,
$$

which holds if and only if $\rho_{A B}=c_{0} \cdot \mathbb{1}_{d}+c_{1} \cdot \rho_{A B}+c_{2} \cdot \rho_{A B}^{2}$. This means that for any eigenvalue $\lambda(\geqslant 0)$ of $\rho_{A B}$ must satisfy that

$$
c_{2} \lambda^{2}+\left(c_{1}-1\right) \lambda+c_{0}=0
$$

Solve this equation, we get

$$
\lambda=\frac{\left(1-c_{1}\right)-\sqrt{\left(1-c_{1}\right)^{2}-4 c_{0} c_{2}}}{2 c_{2}}=\frac{1}{d} .
$$

Note that we have dropped another root being larger than one. Thus $\rho_{A B}$ is maximally mixed state. In fact, we get that $\rho_{A B}=c_{0} \cdot \mathbb{1}_{d}+c_{1} \cdot \rho_{A B}+c_{2} \cdot \rho_{A B}^{2}$ if and only if $\rho_{A B}$ is maximally mixed state. 


\section{References}

[1] S. Bravyi, Compatibility between local and multipartite states, Quant Inf. \& Comput. 4(1), 012-026 (2004).

[2] M-D. Choi, Completely positive linear maps on complex matrices, Linear Alg Appl. 10, 285-290 (1975).

[3] M. Christandl, B. Doran, S. Kousidis, M. Walter, Eigenvalue distributions of reduced density matrices, Comm. Math. Phys. 332, 1-52 (2014).

[4] B. Collins, Moments and Cumulants of Polynomial Random Variables on Unitary Groups, the Itzykson-Zuber Integral, and Free Probability, Int. Math. Res. Not. 17, 953 (2003).

[5] B. Collins, P. Śniady, Integration with Respect to the Haar Measure on Unitary, Orthogonal and Symplectic Group, Comm. Math. Phys. 264(3), 773-795 (2006).

[6] J.P. Dyer, Divergence of Lubkin's series for a quantum subsystem's mean entropy, arXiv:1406.5776

[7] S.K. Foong and S. Kano, Proof of Page's conjecture on the avearge entropy of a subsystem, Phys. Rev. Lett. 72, 1148 (1994).

[8] M. Gessner and H-P Breuer, Generic features of the dynamics of complex open quantum systems: Stataistical approach based on averages over the unitary group, Phys. Rev. E 87, 042128 (2013).

[9] P. Giorda and M. Allegra, Two-qubit correlations revisited: average mutual information, relevant (and useful) observables and an application to remote state preparation, arXiv: 1606.02197

[10] F. Hiai and D. Petz, Introduction to Matrix Analysis and Applications, Hindustan Book Agency, Springer (2014).

[11] S. Jevtic, D. Jennings, and T. Rudolph, Maximally and minimally correlated states attainable within a closed evolving system, Phys. Rev. Lett. 108, 110403 (2012).

[12] S. Jevtic, D. Jennings, and T. Rudolph, Quantum mutual information along unitary orbits, Phys. Rev. A 85, 052121 (2012).

[13] A. Klyachko, Quantum marginal problem and N-representability, J. Phys. Conference Series 36, 72-86 (2006).

[14] A. Lachal, Probabilistic approach to Page's formula for the entropy of a quantum system, Stochasitcs: An International Journal of Probability and Stochastics Processes 78, 157-178 (2006).

[15] E. Lubkin, Entropy of an n-system from its correlation with a k-reservoir, J. Math. Phys. 19(5), 1028 (1978).

[16] K. Modi, M. Gu, Coherent and incoherent contents of correlations, Int. J. Mod. Phys. B 27, 1345027 (2012).

[17] M. Oszmaniec, M. Kuś, Fraction of isospectral states exhibiting quantum correlations, Phys. Rev. A 90, 010302 (2014).

[18] D.N. Page, Average entropy of a subsystem, Phys. Rev. Lett. 71, 1291 (1993). 
[19] S. Sen, Average entropy of a quantum subsystem, Phys. Rev. Lett. 77, 1 (1996).

[20] J. Sánchez-Ruiz, Simple proof of Page's conjecture on the average entropy of a subsystem, Phys. Rev. E 52, 5653 (1995).

[21] M. Walter, B. Doran, D. Gross, and M. Christandl, Entanglement Polytopes: Multiparticle Entanglement from Single-Particle Information, Science 340, 6137 (2013).

[22] L. Zhang, Matrix integrals over unitary groups: An application of Schur-Weyl duality, arXiv:1408.3782v3

[23] L. Zhang, Average coherence and its typicality for random mixed quantum states, J. Phys. A : Math. Theor. to appear

[24] L. Zhang, S-M. Fei, Quantum fidelity and relative entropy between unitary orbits, J. Phys. A : Math. Theor. 47, 055301 (2014).

[25] L. Zhang, L. Chen, and K. Bu, Fidelity between one bipartite quantum state and another undergoing local unitary dynamics, Quant. Inf. Process 14, 4715 (2015).

[26] L. Zhang, U. Singh, and A.K. Pati, Average subentropy, coherence and entanglement of random mixed quantum states, Ann. Phys. 377, 125-146 (2017). 